\title{
An experimental and numerical investigation of a viscoelastic flow around a cylinder
}

\author{
Frank P. T. Baaijens, ${ }^{\text {a) }}$ Hans P. W. Baaijens, Gerrit W. M. Peters, \\ and Han E. H. Meijer \\ Eindhoven University of Technology, Center for Polymers and \\ Composites, P.O. Box 513, 5600 MB Eindhoven, The Netherlands
}

(Received 3 April 1993; accepted 7 September 1993)

\begin{abstract}
Synopsis
In this paper the plane flow of a shear thinning solution of 5 wt\% polyisobutylene (PIB) in tetradecane (C14) (PIB/C14) around a cylinder placed between parallel plates is investigated numerically and experimentally. Both Laser Doppler Anemometry and Flow Induced Birefringence measurements are performed and compared with numerical results, using a Discontinuous Galerkin method and a one-mode Phan-Thien Tanner model. Previous work has shown a good agreement between measured and computed stress fields for the flow through a planar four-to-one contraction with this fluid. The flow around a cylinder, however, leads to some surprising differences.
\end{abstract}

\section{INTRODUCTION}

Without exception, constitutive parameters of polymer melts and solutions are determined via well-defined viscometric shear flows. It is generally assumed that constitutive equations thus characterized also hold in situations where the material is subjected to a variety of complicated combined shear and elongational flows existing in many practical processes.

Viscometric flows, although simple from a theoretical point of view, may be difficult to realize practically [e.g., extensional flows, Walters (1992) and Hudson and Jones (1993)], and only give information in a very limited flow regime. The use of both Laser Doppler Anemometry (LDA) and Flow Induced Birefringence (FIB) measurements in combination with robust numerical algorithms for viscoelastic flows, today allows the detailed evaluation of the performance of constitutive equations in complex flows. It is expected that combined velocity-stress measurements of complex, multidimensional flows may provide new insight in the constitutive behavior of polymeric liquids, leading to improved constitutive models and better qualitative predictive capabilities of numerical simulations in practically relevant situations. Ultimately, use of complex flow data may even prove to be essential to properly characterize the parameters of viscoelastic material parameters. A prerequisite for such an approach is the availability of good experimental facilities, a reliable numerical code, and constitutive models that cover a wide range of flows.

Armstrong et al. (1992) and Quinzani (1991), have investigated the flow of a shear thinning solution of $5.0 \mathrm{wt} \%$ polyisobutylene (PIB) in tetradecane (C14), PIB/C14,

8) Also at Philips Research Laboratories, P.O. Box 80000, 5600 JA Eindhoven, The Netherlands. 
through a planar four-to-one contraction. By measuring velocities and stresses along the centerline of the four-to-one contraction, Armstrong et al. (1992) tested the performance of six selected constitutive models (the Giesekus, Bird-DeAguir, PhanThien Tanner, Acierno et al., White-Metzner, and the Oldroyd-B model) in a strong elongational flow. They concluded that the PTT model gave the best overall performance.

Subsequently, Baaijens (1993) showed that reasonable agreement between measured, Armstrong et al. (1992) and Quinzani (1991), and computed stress fields is obtained for this contraction flow using a single- or multimode Phan-Thien Tanner (PTT) model.

The key purpose of this paper is to investigate if the one-mode PTT model performs equally well (for this shear thinning PIB/C14 solution) in predicting stress fields for the flow around a cylinder placed between two parallel plates. Detailed velocity (LDA) and stress (FIB) measurements are compared with numerical predictions. McKinley (1991) has also investigated the flow around a cylinder, but with a different fluid, a constant viscosity solution of polyisobutylene and polybutene in tetradecane (PIB/PB/C14), and only velocity measurements were performed. According to McKinley (1993), FIB measurements on the PIB/PB/C14 are practically impossible. Rajagopalan et al. (1992) performed FIB measurements on the eccentric rotating cylinder problem, with a solution based on a polystyrene in tricresyl phosphate, and no pointwise velocity measurements are performed. Hence, the current work is unique, in the sense that the flow is investigated experimentally, by measuring both velocities and stresses, and numerically simultaneously in a geometry-fluid combination not investigated before. This allows an immediate evaluation of the performance of the PTT model in a complicated flow situation.

The numerical scheme applied is the Discontinuous Galerkin (DG) method originally proposed for viscoelastic flows by Fortin and Fortin (1990) and further refined by Fortin et al. (1992). However, a slightly different implementation is adopted in this work.

In this paper, first of all, the material characterization is discussed. A one-mode PTT model fit is obtained based on steady- and small-amplitude oscillatory shear flow. Thereafter, the experimental procedure to measure velocities and stresses is discussed in detail. The model problem and numerical solution method are discussed next, whereafter numerical and experimental results are pointwise compared at a number of locations in the flow geometry. The discrepancies between predictions and experimental observations are discussed in the final section, clearly indicating the failure of the Phan-Thien Tanner model to capture the complicated stress distribution in the wake of the cylinder.

\section{MATERIAL CHARACTERIZATION}

The $5 \mathrm{wt} \% \mathrm{PIB} / \mathrm{Cl} 4$ solution was prepared by dissolving polyisobutylene VistanexL120 (Exxon Chemical) in C14. The material is rheologically characterized on an RFS-2 Mechanical Spectrometer (Rheometrics, Inc.) in a cone-plate configuration in both steady- and small-amplitude oscillatory shear flow. Steady shear data was obtained at $293 \mathrm{~K}$ over a shear rate range of $10^{-1}$ to $300\left(\mathrm{~s}^{-1}\right)$. Oscillatory data are obtained in a temperature range from 278 to $317 \mathrm{~K}$, and time-temperature superposition is used to obtain a master curve.

Figure 1 shows the master curves for $\eta^{\prime}$, the loss angle as a function of frequency, and the steady shear viscosity as a function of the shear rate. Only about $10 \%$ of the 

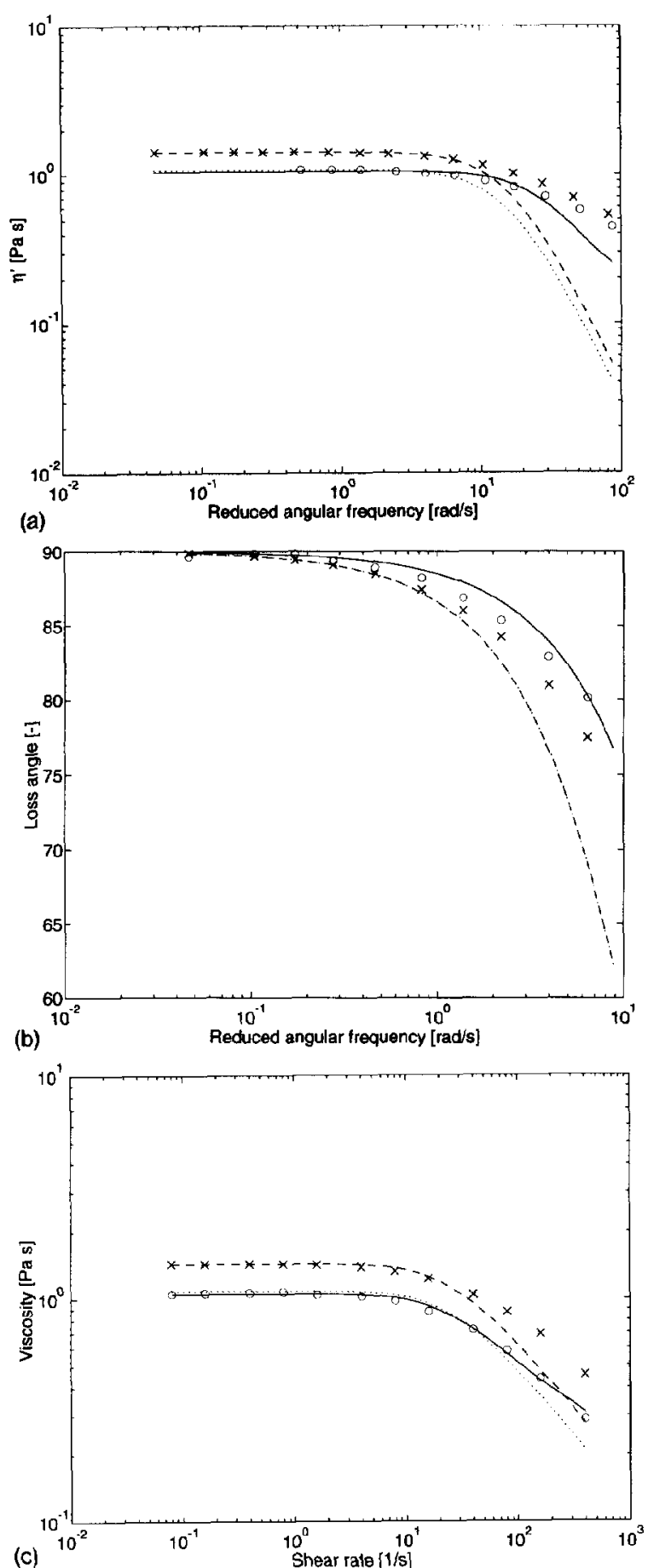

FIG. 1. Fits on PIB/C14 data at a reference temperature of $297 \mathrm{~K}$. (a) $\eta^{\prime}$, (b) loss angle, and (c) steady-state viscosity. The open symbols denote the measured data of the current test fluid, the crosses denote the data of Armstrong et al. (1992), the solid line refers to the EUT fit, the dashed line to the MIT fit, and the dotted line to the EUT/MIT fit, all of Table I. Remark: only about $10 \%$ of the actual data points are shown. 
data points are shown, to maintain a good visibility of the various curves. An Arrhenius fit, $\ln \left(a_{T}\right)=2605\left(1 / T-1 / T_{0}\right), T_{0}=297.7 \mathrm{~K}$ is used to model the temperature dependency of vertical shift factor, as shown in Fig. 2.

The polymer contribution of the one-mode PTT model $\tau$ to the total extra stress is given by

$$
\frac{\partial \tau}{\partial t}+\mathbf{u} \cdot \nabla \boldsymbol{\tau}-\mathbf{L} \cdot \boldsymbol{\tau}-\boldsymbol{\tau} \cdot \mathbf{L}^{T}+\left(\frac{1}{\lambda}+\frac{\epsilon}{\eta} \operatorname{tr}(\boldsymbol{\tau})\right) \boldsymbol{\tau}=\frac{2 \eta}{\lambda} \mathbf{D},
$$

where $u$ denotes the velocity field, $\lambda$ is the relaxation time, $\eta$ is the viscosity, and $\epsilon$ is an adjustable parameter that, in particular, affects the elongational and steady shear behavior of the model. The current value is based on a fit on the steady shear viscosity curve, as no a priori elongational data is available. The solvent viscosity (of the $\mathrm{C14}$ solvent) is denoted by $\eta_{0}$.

Figure 1 shows a number of one-mode PTT model fits on either the current data, or on the MIT data given by Quinzani et al. (1990). The open symbols are obtained in our laboratory, while the crosses denote the MIT data. The most significant difference is found in the zero-shear rate viscosity. The MIT fluid has a zero-shear rate viscosity of $1.426 \mathrm{~Pa} \mathrm{~s}$, while the EUT fluid has a viscosity of $1.053 \mathrm{~Pa}$ s. It is not known what causes this discrepancy.

Fitting the data is not without ambiguity, as is shown next.

Fitting a one-mode PTT model on $\eta^{\prime}$, the loss angle and the viscosity curves simultaneously produces the solid lines in Fig. 1. This fit is referred to as the EUT fit in Table I. Clearly, in this case $\eta_{0}$ cannot be interpreted as the solvent viscosity, which for $\mathrm{C} 14$ equals $0.002 \mathrm{~Pa}$ s.

The one-mode MIT fit given by Armstrong et al. (1992) is denoted by the dashed lines. Now, choosing $\eta_{0}, \epsilon$, and $\lambda$ equal to the MIT fit, and only adjusting the viscosity

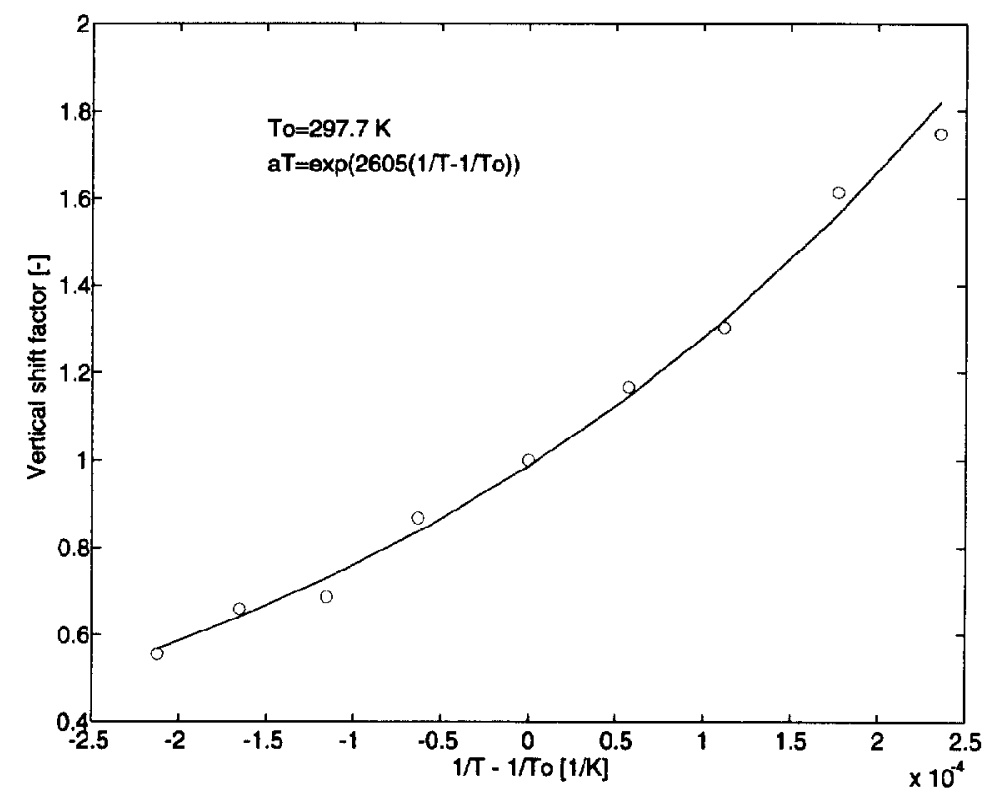

FIG. 2. Vertical shift factor $a_{T}$ as a function of temperature. 
TABLE I. One-mode fits on current data and fit of Armstrong et al. (1992).

\begin{tabular}{lllll}
\hline \hline \multicolumn{1}{c}{ Identifier } & $\lambda(\mathrm{s})$ & $\eta$ (Pa s) & \multicolumn{1}{c}{$\epsilon$} & $\eta_{0}$ (Pa s) \\
\hline EUT fit & 0.0312 & 0.9103 & 0.4461 & 0.1426 \\
EUT/MIT fit & 0.06 & 1.0807 & 0.1 & 0.002 \\
MIT fit & 0.06 & 1.424 & 0.1 & 0.002 \\
\hline \hline
\end{tabular}

$\eta$, the dotted lines in Fig. 1 are obtained. This fit is referred to as the EUT/MIT fit in Table I. The EUT/MIT and the MIT fit are of equal quality, with respect to their data points, while for the loss angle both fits coincide.

Inspection of Fig. 1 suggests that the EUT fit is somewhat better than the EUT/ MIT fit, provided that the loss of interpretation of $\eta_{0}$ is accepted. In the subsequent computations, both the EUT and the MIT fit are used. The EUT/MIT fit produced results that are only marginally different from the MIT fit results, and are not discussed in the sequel.

\section{EXPERIMENTAL METHODS}

Two experimental techniques, Laser Doppler Anemometry (LDA) and a Flow Induced Birefringence (FIB) technique, have been used for measuring velocities and stresses, respectively. The LDA and FIB measurements have been performed sequentially on the same experimental setup. In this section, first the flow loop and then the LDA and the FIB systems are explained.

\section{A. The flow loop}

The flow loop, drawn schematically in Fig. 3, is used during the experiments. A gear pump (Verder) (1) forces the PIB/C14 solution from the 2.51 glass container (2) through a tube into the flow cell (3). This flow cell is made of PMMA. The steel cylinder (4) has a radius $R=3.75 \mathrm{~mm}$, and is positioned symmetrically between the two large sidewalls. The width $w$ of the flow channel is $15.2 \mathrm{~mm}$ and the depth $d$ is 118 $\mathrm{mm}$. The ratio $2 R / w$ is 0.49 . The width-depth ratio is $1: 7.8$, to effect a twodimensional, planar flow near the centerline of the flow cell. In the sidewalls, $8 \mathrm{~mm}$ thic:. windows (5) of borosilicate crown glass (BK7) were made to reduce parasitic birefringence. These windows were sealed with silicon kit and clamped tight with two clamps. Close to the bottom of the flow cell, a narrow slit (6) just above the two inlet nozzles smoothes the inflow velocity distribution. During the experiments an amount of approximately $2.51 \mathrm{PIB} / \mathrm{C} 14$ solution was used, with an average velocity in the flow domain of $26 \mathrm{~mm} / \mathrm{s}$. All measurements were performed at a temperature $T=23$ $\pm 2{ }^{\circ} \mathrm{C}$.

\section{B. Laser Doppler Anemometry}

Laser Doppler Anemometry is a technique to measure pointwise fluid velocities with a high spatial resolution [e.g., Drain (1980)]. The LDA system uses fiber optics and operates in a backscatter mode. The $300 \mathrm{~mW}$ Ar-ion laser (Ion Laser Technology $5500 \mathrm{~A})$ generates laser light at three different wavelengths. Each color is divided in two beams, one of which is frequency shifted by a Bragg cell. A power control unit enables us to operate the laser with a variable power between 0 and $300 \mathrm{~mW} ; 150 \mathrm{~mW}$ sufficed in most cases. Glass fibers transmit the laser beams to the measuring probe. A front lens with a focal length of $80 \mathrm{~mm}$ focuses the beams in a measuring volume, with 


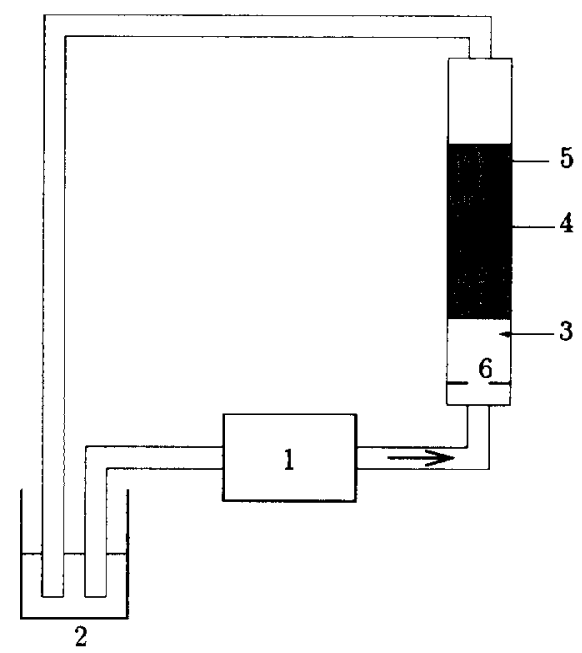

FIG. 3. Schematic drawing of the flow loop. 1: Gear pump; 2: glass container; $3:$ flow cell; 4 : steel cylinder; 5: borosilicate crown glass windows; 6 : slit.

dimensions $50 \times 50 \times 200 \mu \mathrm{m}$. The part of the backscattered signal, which is focused in the measuring probe, is transmitted to the Flow Velocity Analyzer (FVA, Dantec $58 \mathrm{~N} 20)$ by fiber. Only the green beams $(\lambda=514.5 \mathrm{~nm})$ were used in this experiment.

As seeding a small amount of Iriodine (Iriodine 111, Merck) particles has been dispersed in the PIB/C14 solution. Iriodine consists of highly reflective aluminum plates, with a diameter of $2-50 \mu \mathrm{m}$. A concentration of approximately $0.01 \mathrm{~g} / 1$ Iriodine proved to be sufficient. The resolution of the velocity measurements is mainly restricted by the analyzing hardware, and is approximately $0.5 \mathrm{~mm} / \mathrm{s}$. The measuring probe is mounted on a traversing system, which has a resolution of approximately 0.05 $\mathrm{mm}$. Data acquisition and processing is performed on a PC with FLO ware (Dantec).

\section{Flow Induced Birefringence}

\section{Stress optic rule}

Flow Induced Birefringence enables measurement of extra stresses in many flowing materials, especially in polymer melts and solutions. For many polymeric materials, extensive studies [e.g., Wales (1976)] have shown that the refractive index tensor is related to the extra stress tensor by a simple linear relation [Fuller (1990)], the stress optical rule:

$$
n_{i j}=C \tau_{p, i j}+n_{m} \delta_{i j},
$$

where $n_{i j}$ is the refractive index tensor, $\tau_{p, i j}$ is the polymer contribution to the extra stress tensor, $C$ is the stress-optical coefficient, $\delta_{i j}$ is the unity tensor, and $n_{m}$ is the mean refractive index. This rule has been found to be widely valid for most concentrated polymer solutions and melts. In case the orientation of chain segments reaches saturation, or light scattering dominates, the rule will break down. The PIB/C14 fluid has a stress optic coefficient equal to $1.87 \times 10^{-9} \mathrm{~Pa}^{-1}$ [Quinzani (1991)].

Thus, polymer stresses $\tau_{p, i j}$ are coaxial with refractive indices $n_{i j}$, and the eigenvalues of both tensors are proportional. The stress optical rule can be rewritten as 


$$
\Delta n_{i}=C \Delta \tau_{p, i}
$$

with $\Delta n_{i}$, the $i$ th principle refractive index difference and $\Delta \tau_{p, i}$ the $i$ th principle stress difference. The local principle axis frames have an orientation angle $\chi$ with the fixed laboratory frame. Transformed to this fixed coordinate frame, the shear stress $\tau_{p, x y}$ and the first normal stress difference $\tau_{p, x x}-\tau_{p, y y}$ are related to the orientation $\chi$ and the birefringence $\Delta n$ by

$$
\begin{gathered}
\tau_{p, x y}=\frac{\Delta n \sin 2 \chi}{2 C}, \\
\tau_{p, x x}-\tau_{p, y y}=\frac{\Delta n \cos 2 \chi}{C} .
\end{gathered}
$$

\section{Measurement technique}

Flow Induced Birefringence has been used in numerous studies to measure stress fields, in particular, in polymer melts, as reviewed, for example, by Mackay and Boger (1988) and Quinzani (1991). Pointwise measurements of birefringence were improved by the introduction of a two color flow birefringence technique [Chow and Fuller (1984)], and of rotating polarization modulation techniques [Fuller and Mikkelsen (1989); Fuller (1990)].

The system used in this study is based on the latter principle.

Figure 4 shows schematically the FIB system. The light source (1) is a $5 \mathrm{~mW}$ He-Ne laser (Spindler and Hoyer). The red laser beam $(\lambda=632.8 \mathrm{~nm})$ is focused with a $200 \mathrm{~mm}$ lens, resulting in a beam with a waist of $0.20 \mathrm{~mm}$, and with a diameter of $0.28 \mathrm{~mm}$ at the entrance of the flow cell. The beam passes the polarizer (2), that is oriented at an angle of $0^{\circ}$ with the optical axis of the system (defined as the vertical axis), and crosses successively the rotating half-wave plate (Rheometrics, Inc.) (3), the flow cell (4) with the birefringent medium, the quarter-wave plate (at $45^{\circ}$ with the optical axis) (5), and the analyzer (at $\left.0^{\circ}\right)(6)$. Finally, the intensity of the beam is measured by the detector.

The polarization state of the detected light can be calculated by use of Jones matrices. The intensity $I$ on the detector is given by the expression

$$
I=I_{m}+I_{\text {in }}+I_{\text {out }},
$$

with

$$
\begin{gathered}
I_{\text {in }}=R_{1} \sin \omega t, \\
I_{\text {out }}=R_{2} \cos \omega t,
\end{gathered}
$$

and

$$
\begin{gathered}
R_{1}=I_{m} \cos 2 \chi \sin \delta, \\
R_{2}=-I_{m} \sin 2 \chi \sin \delta,
\end{gathered}
$$

where $I_{m}$ is the mean intensity; $I_{\text {in }}$ is the in-phase (with the rotating half-wave plate) intensity; $I_{\text {out }}$ is the out-phase intensity; $\omega$ is the angular frequency of the rotating half-wave plate; and $t$ is the time.

The lock-in amplifier (EG\&G Princeton Applied Research, model 5210) measures the in- and out-phase components of the intensity signal, using the separately mea- 


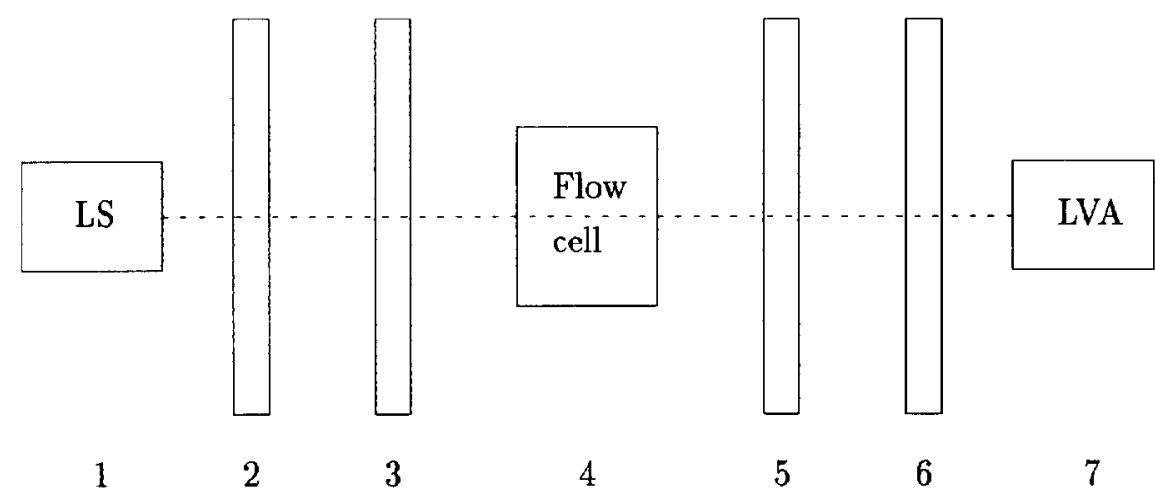

FIG. 4. The FIB measuring system, based on polarization modulation with a rotating half-wave plate. 1: Light source; 2: polarizer; 3 : half-waye plate; 4: flow cell; 5 ; birefringent medium; 6: analyzer.

sured reference frequency $\omega$. The intensity signal is monitored on an oscilloscope, and the time-averaged mean intensity $I_{m}$ is measured using a simple electronic circuit. Data acquisition is performed on a PC.

\section{Calculation of stresses}

From the measured intensities $I_{m}, I_{\text {in }}$, and $I_{\text {out }}$, the angle $\chi$ and retardation $\delta$ can be calculated using

$$
\begin{aligned}
& \tan 2 \chi=-\frac{\left\|R_{1}\right\|}{\left\|R_{2}\right\|}, \\
& \sin ^{2} \delta=\frac{I_{\text {in }}^{2}+I_{\text {out }}^{2}}{I_{m}^{2}} .
\end{aligned}
$$

Once the angles $\chi$ and $\delta$ are known, the shear stress $\tau_{x y}$ and normal stress difference $\tau_{x x}-\tau_{y y}$ follow from Eqs. (4) and (5).

Problems arise in these calculations when $\delta$ is outside the interval $[0, \pm 2 \pi]$, but in our experiments $\delta$ is very small. In case $|\chi|$ becomes larger than $\pi / 4, \tan 2 \chi$ changes sign. This causes an unrealistic jump in the calculated $\chi$ when applying the arctangents. Corrections for this have been made by adding $\pm \pi / 2$ to $\chi$ when necessary.

During the experiments, it was found that the birefringence of the glass windows affected the measured signal. To eliminate this effect, the following procedure has been adopted. First, on all measuring points the intensity signals were measured during zero flow at ambient pressure. Then the fluid was pumped through the system and when steady state was reached the intensity signals were measured again on the same points. With $\delta_{\text {glass }}$ the retardation of the glass windows, the following approximations are made: $\cos \delta_{\text {glass }}=1, \sin \delta_{\text {glass }} \sin \delta=0$, and $\cos \delta=1$. All these approximations are legitimate because both $\delta_{\text {glass }}$ and $\delta$ are very small ( $\leqslant 0.2 \mathrm{rad}$ ). These approximations allow us to calculate the stresses of the polymer after subtracting the in- and out-phase intensities measured during zero flow from their values measured during flow.

During flow, the pressure in the flow cell rises to $\mathscr{O}\left(10^{3}\right) \mathrm{Pa}$. Parasitic birefringence due to this pressurization, taking account of both tensile and bending phenomena, is 
computed to be of $\mathscr{O}\left(10^{-10}\right)$, while the birefringence induced by the flowing medium of interest here is larger than $\mathscr{O}\left(10^{-8}\right)$. Hence this parasitic birefringence may be neglected.

\section{PROBLEM DEFINITION}

In the numerical analysis the following problem is studied. Consider the steady, inertialless flow of a PTT fluid in a two-dimensional domain $\Omega$ with boundary $\Gamma$, defined by the following.

Problem 1 (PVE): Given $\mathbf{u}^{0}: \Gamma_{u} \rightarrow \mathbb{R}^{2}$ and $\mathrm{t}^{0}: \Gamma_{\tau} \rightarrow \mathbb{R}^{2}$, find the plane stress field $\tau(\mathrm{x})$ : $\Omega \mapsto \mathbb{R}^{(2 \times 2)}$, the velocity field $\mathbf{u}(\mathbf{x}): \Omega \mapsto \mathbb{R}^{2}$ and the pressure field $p(\mathbf{x}): \Omega \mapsto \mathbb{R}$ for all $x \in \Omega$, such that

$$
\begin{gathered}
\boldsymbol{\nabla} \cdot\left(-p \mathbf{I}+2 \eta_{0} \mathbf{D}+\boldsymbol{\tau}\right)=\mathbf{0}, \\
\boldsymbol{\nabla} \cdot \mathbf{u}=0, \\
\mathscr{L} \boldsymbol{\tau}-\frac{2 \eta}{\lambda} \mathbf{D}=\mathbf{0},
\end{gathered}
$$

with

$$
\mathscr{L} \tau=\mathbf{u} \cdot \nabla \tau-\mathbf{L} \cdot \tau-\tau \cdot \mathbf{L}^{T}+\left(\frac{1}{\lambda}+\frac{\epsilon}{\eta} \operatorname{tr}(\tau)\right) \tau,
$$

with $\mathbf{L}=(\boldsymbol{\nabla u})^{c}$ and $\mathbf{D}=\frac{1}{2}\left(\mathbf{L}+\mathbf{L}^{\mathrm{T}}\right)$, while the following boundary conditions are specified on $\Gamma$ :

$$
\begin{gathered}
\mathbf{u}(\mathbf{x})=\mathbf{u}^{0}(\mathbf{x}), \quad \text { on } \Gamma_{u}, \\
{\left[-p \mathbf{I}+2 \eta_{0} \mathbf{D}+\tau(\mathbf{x}, t)\right] \cdot \mathbf{n}=\mathbf{t}^{0}(\mathbf{x}, t), \text { on } \Gamma_{\tau},}
\end{gathered}
$$

while at the inflow boundary,

$$
\tau=\tau^{0}, \text { on } \Gamma_{\text {in }},
$$

with $\Gamma=\Gamma_{u} \cup \Gamma_{\tau} \cup \Gamma_{\text {in }}$, $n$ is the unit outward normal at $\Gamma$, and $\Gamma_{\text {in }}$ is the inflow boundary: the part of $\Gamma$ where $u \cdot n<0$.

In all cases, a fully developed Poiseuille flow is assumed at the inflow boundary.

\section{NUMERICAL METHOD}

Although the operator-splitting based method used in Baaijens (1993) gave good agreement with the experimental results of Armstrong et al. (1992), the Discontinuous Galerkin (DG) method of Fortin and Fortin (1990) is used here, because, in this particular case, the DG method turned out to be computationally more efficient. This is due to the use of only a one-mode version of the PTT model at moderate values of the Deborah number at stationary flow conditions. Assuming the extra stresses to be interpolated discontinuously at the element borders from the onset, the DG method is defined by the following.

Problem 2 (DG): Find $(\tau, \mathrm{u}, p)$, such that for all $(\mathrm{s}, \mathrm{v}, q)$;

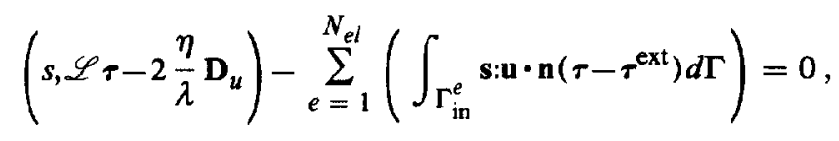




$$
\begin{gathered}
-\left(\mathbf{D}_{v}, 2 \eta_{0} \mathbf{D}_{u}+\tau\right)+(\boldsymbol{\nabla} \cdot \mathbf{u}, p)=0, \\
(q, \boldsymbol{\nabla} \cdot \mathbf{u})=0 .
\end{gathered}
$$

In the above, $(\cdot, \cdot)$ and $(\cdot, \cdot)_{e}$ denote the appropriate inner product on $\Omega$ and $\Omega^{e}$, respectively, $\Gamma_{\text {in }}^{e}$ is the inflow boundary of element $\Omega^{e}, \mathbf{n}$ is the unit outward normal to $\Gamma^{e}, \tau^{\text {ext }}$ is the stress tensor of the neighboring, upwind, element. Furthermore, $\mathbf{D}_{u}=\frac{1}{2}\left[\nabla \mathbf{u}+(\nabla \mathbf{u})^{T}\right]$ and $\mathbf{D}_{v}=\frac{1}{2}\left[\nabla \mathbf{v}+(\nabla \mathbf{v})^{T}\right]$.

Usually, a Picard type of iteration scheme or a decoupled solution process, in conjunction with the GMRES solver is used for the DG method [see Fortin and Fortin (1991) and Fortin et al. (1992)]. In this paper, however, a Newton-like iteration scheme is used. The method cannot be fully Newton due to the nondifferentiability of the boundary integral over $\Gamma_{\text {in }}$; nevertheless, a quadratic rate of convergence is retained in all cases investigated here. The resulting set of equations is solved using a GMRES algorithm.

In this work the extra stresses are discretized with a piecewise discontinuous biquadratic Lagrange polynomial, the velocities with its continuous counterpart, and the pressure is discretized with a piecewise discontinuous linear polynomial: $(\mathrm{s}, \mathrm{u}, p) \rightarrow Q_{2}^{d} Q_{2} P_{1}^{d}$.

\section{COMPARISON OF NUMERICAL AND EXPERIMENTAL RESULTS}

The flow domain used in the numerical analysis covers the $x / R$ domain $x / R \in$ $[-16,16]$, with $R=3.75 \mathrm{~mm}$ the radius of the cylinder. The channel height $H=7.5$ $\mathrm{mm}$, hence the ratio $H / R=2$. The Deborah number is defined as

$$
\mathrm{De}=\frac{\lambda\langle v\rangle}{R},
$$

where $\langle v\rangle$ is the average upstream velocity. In the experiments, $\mathrm{De}=\mathbf{0 . 2 1 6}$. For the shear rates employed in this study, the above Deborah number virtually coincides with a shear-dependent Deborah number given by $\mathrm{De}=\Psi_{1}(\dot{\gamma}) \dot{\gamma} / 2 \eta(\dot{\gamma})$.

Outline of the results. At a number of cross sections, $x / R=0,1.5,2,3.5$, and 5 , as well as along the symmetry line FIB measurements are performed. The axial velocity field is only measured at $x / R=-20,0,1.5$ and along the symmetry line. Results are compared in Figs. 5-10. In all cases the open symbols denote the measured results, the solid line is obtained using the one-mode EUT fit (see Table I), the dashed line represents a polynomial fit of the measured data, and the dash-dotted lines are obtained using the MIT-fit parameters (see Table I), giving a Deborah number of 0.416 . The measured $N_{1}$ distribution behind the cylinder for $x / R \in[1.5,5]$, and $y / R \in$ $[-1.8,1.8]$ is depicted in Fig. 11(b). The surface is obtained by means of a polynomial fit, while the open symbols denote the actual data points. Figure 11(c) shows the computed $N_{1}$ distribution in that area, while Fig. 12 shows the computed $N_{1}$ distribution for $x / R \in[-5,5]$. Figure 13 shows the effect of a change in relaxation time upon using the MIT-fit parameters on the first normal stress coefficient.

Velocity data. The measured velocities compare quite well with the predicted data, and little difference is found between the computational predictions based on different parameter fits.

Along the inflow boundary, see Fig. 5, the velocity profile is Newtonian, and no visible difference exists between the computed profiles. The discrepancy between predicted and computed values is due to a slight difference in experimental and numerical 

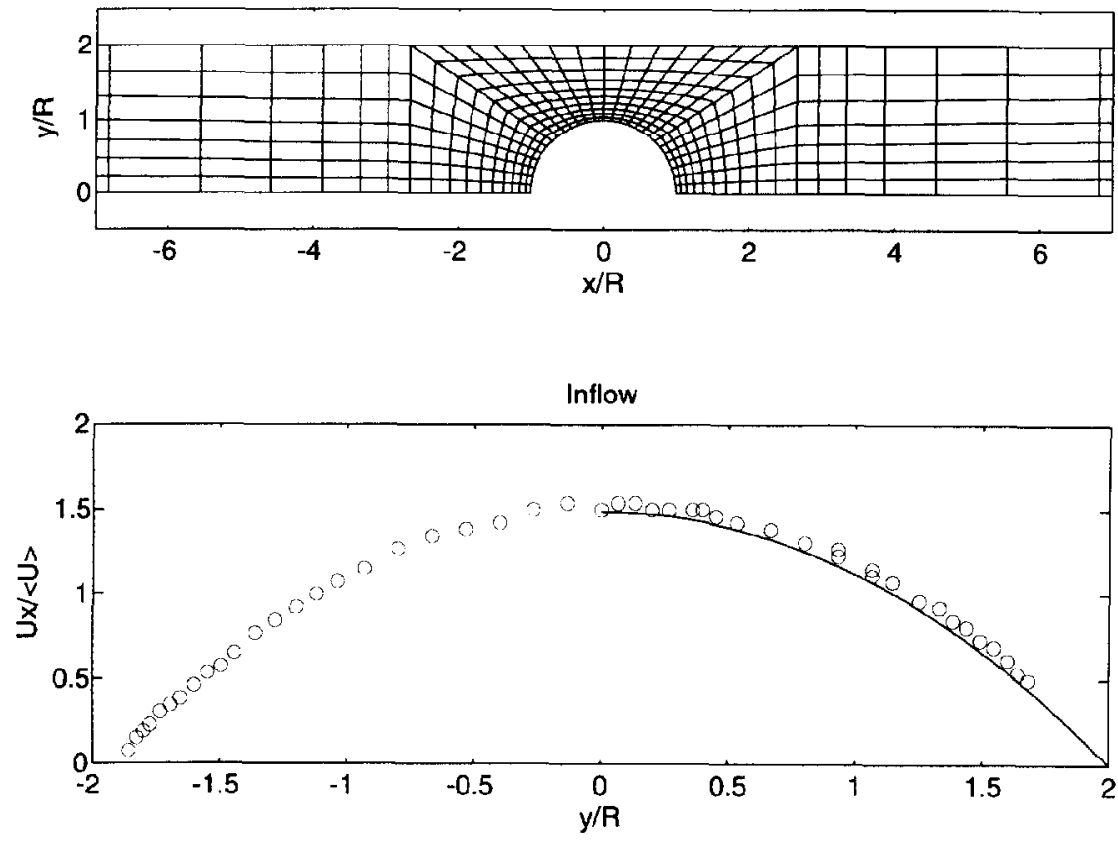

FIG. 5. Mesh used in the computations and measured (open symbols) and computed (solid and dashdotted line) inflow velocity profile. The solid line (based on EUT-fit parameters) and the dash-dottcd line (based on MIT-fit parameters) coincide.
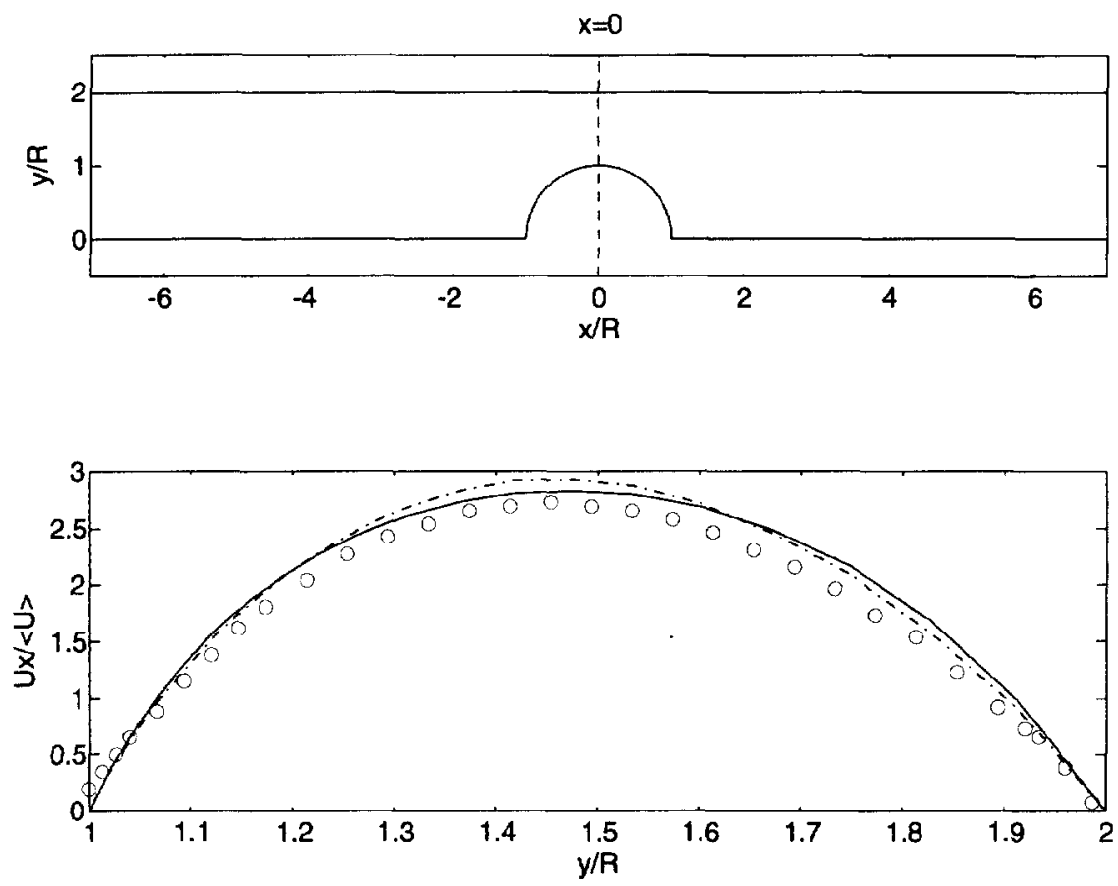

FIG. 6. Comparison of measured (open symbols) and computed velocity $u_{x}$ at $x=0$. (Solid line: based on EUT fit; and dash-dotted line based on MIT-fit parameters). 

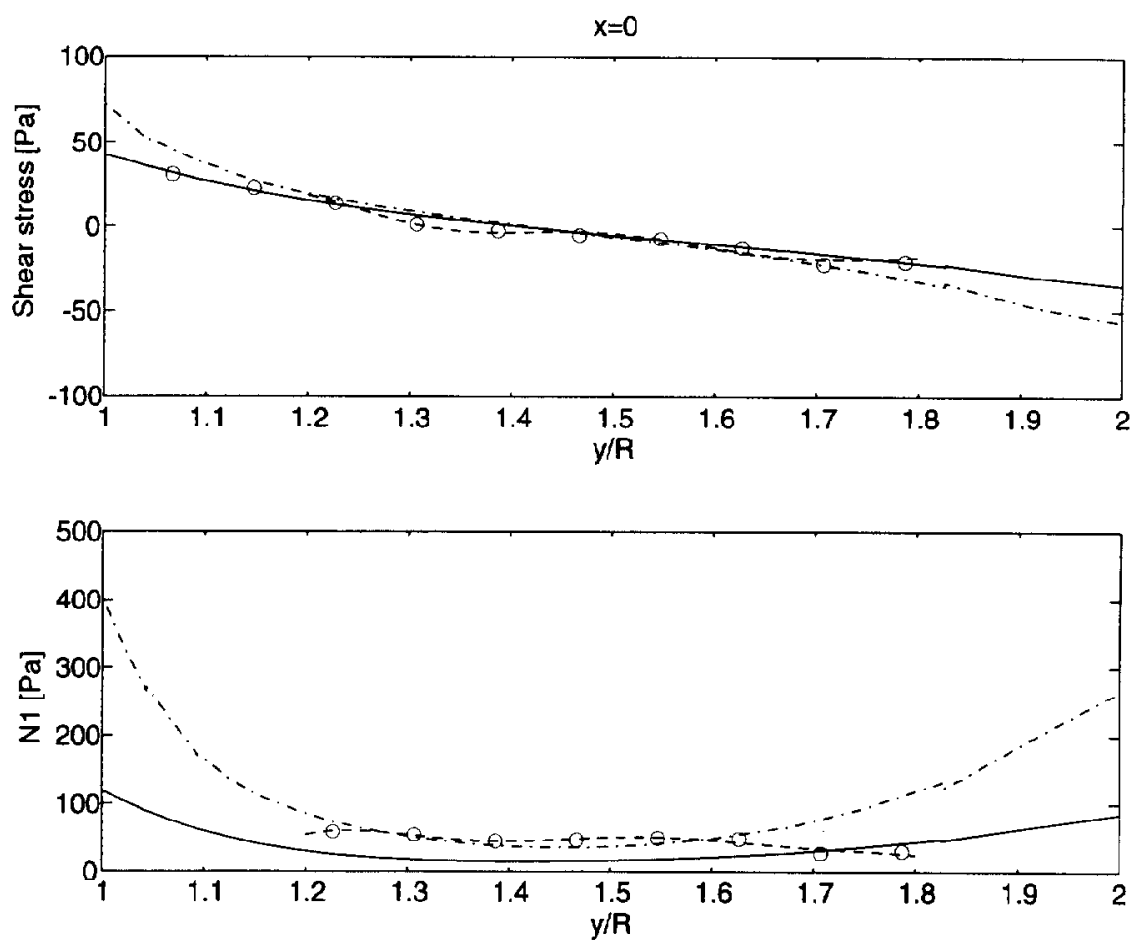

FIG. 7. Comparison of the first normal stress difference $N_{1}$ and the shear stress at $x=0$.

flow rate. Velocity data is not collected at the same time, and a variation of 5\% in flow rate in between the experiments is noticed.

The difference between experimental and numerical velocity profile at the $x / R=0$ cross section, see Fig. 6, is attributed to a slight eccentricity of the cylinder with respect to the two parallel plates. Further, a small, but noticeable, difference exists between the computed curves, clearly indicating that the velocity is affected by viscoelasticity. Shear rates at the cylinder wall are of $\mathscr{O}\left(100 \mathrm{~s}^{-1}\right)$, hence the fiuid is obviously in the shear thinning region.

Along the symmetry line, good agreement between predicted and measured velocity profiles is observed, and little difference between the computed curves is noticed: see Fig. 10. This despite the strong differences between the predicted stress profiles. A closer look at the velocity profile at the wake of the cylinder, Fig. 14, shows that the experimental velocity rise is somewhat slower than the computed value. A slightly slower velocity rise at the wake of the cylinder compared to the Newtonian case is consistent with the results of McKinley (1991), Fig. 7.6, but this does not explain the difference between the measured and computed viscoelastic velocity profile. This result may indicate that there is a small offset error in the experimental $x$ ordinate of about $\Delta x / R=0.1$, as a negative shift of this amount virtually removes the discrepancy observed. However, this shift cannot be completely traced back in the experimental system. The traversing system has an accuracy of $0.05 \mathrm{~mm}$, and the offset error in defining the position of the cylinder wall is expected to be less than $\Delta x / R=0.02$. More likely, a combination of this small offset error, and an uncertainty of the flow rate, as mentioned above, accounts for the differences found. Part of the differences 

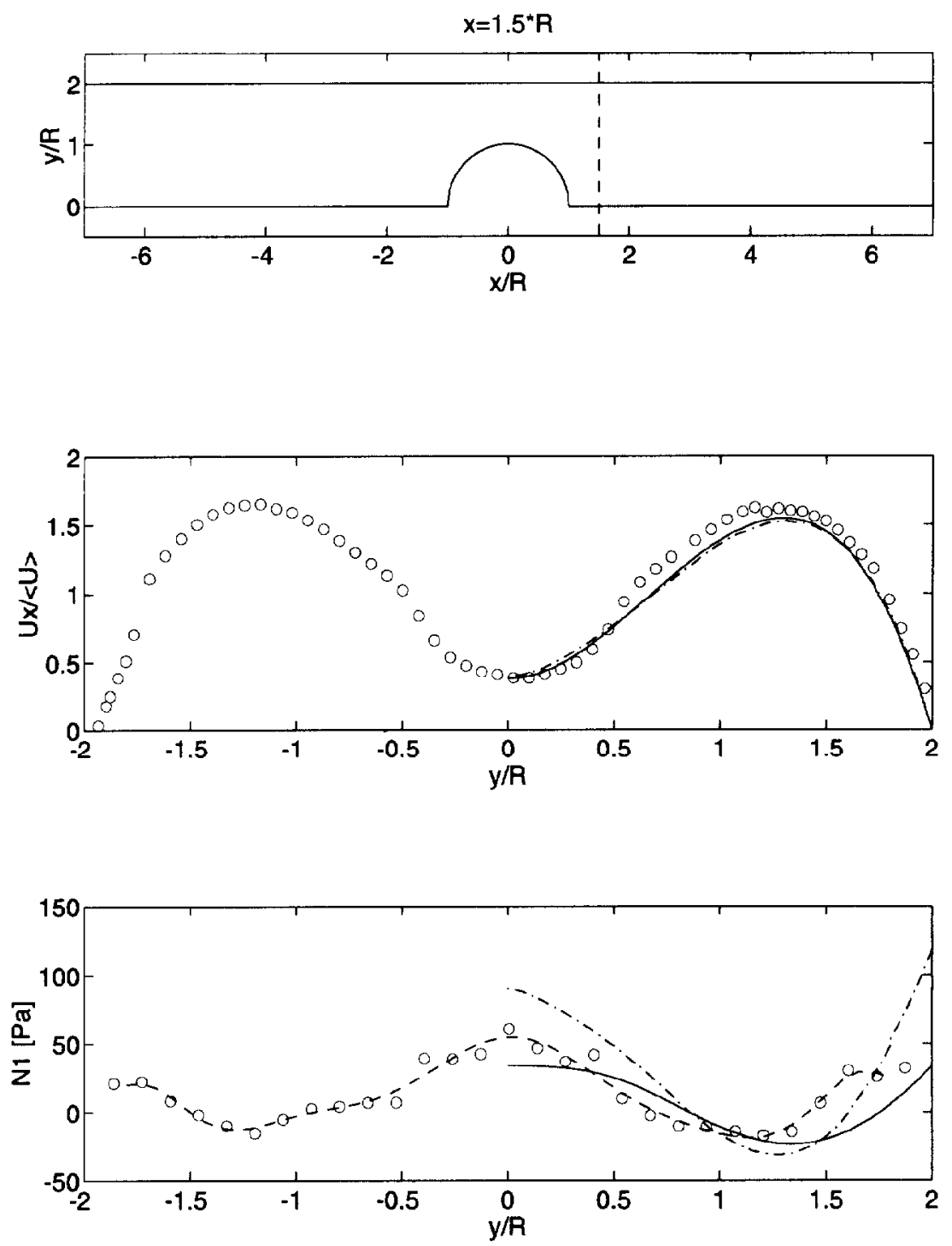

FIG. 8. Comparison of velocity $u_{x}$ and first normal stress difference $N_{1}$ at $x=1.5 R$. The dashed line represents a polynomial fit through the data points indicated by the open symbols. The solid lines represent the computed results based on the EUT-fit parameters and the dash-dotted lines are based on the MIT-fit parameters.

found at $x / R=1.5$ may also be attributed to this, though some effect of viscoelasticity may also be expected, as is indicated by the differences in computed values. In particular, at this cross section, a small offset error of the $x$ ordinate may cause significant changes in the measured velocity profiles as high velocity gradients in the $x$ direction exist in this area.

Stress data. A dramatic discrepancy between predicted and measured stress profiles 

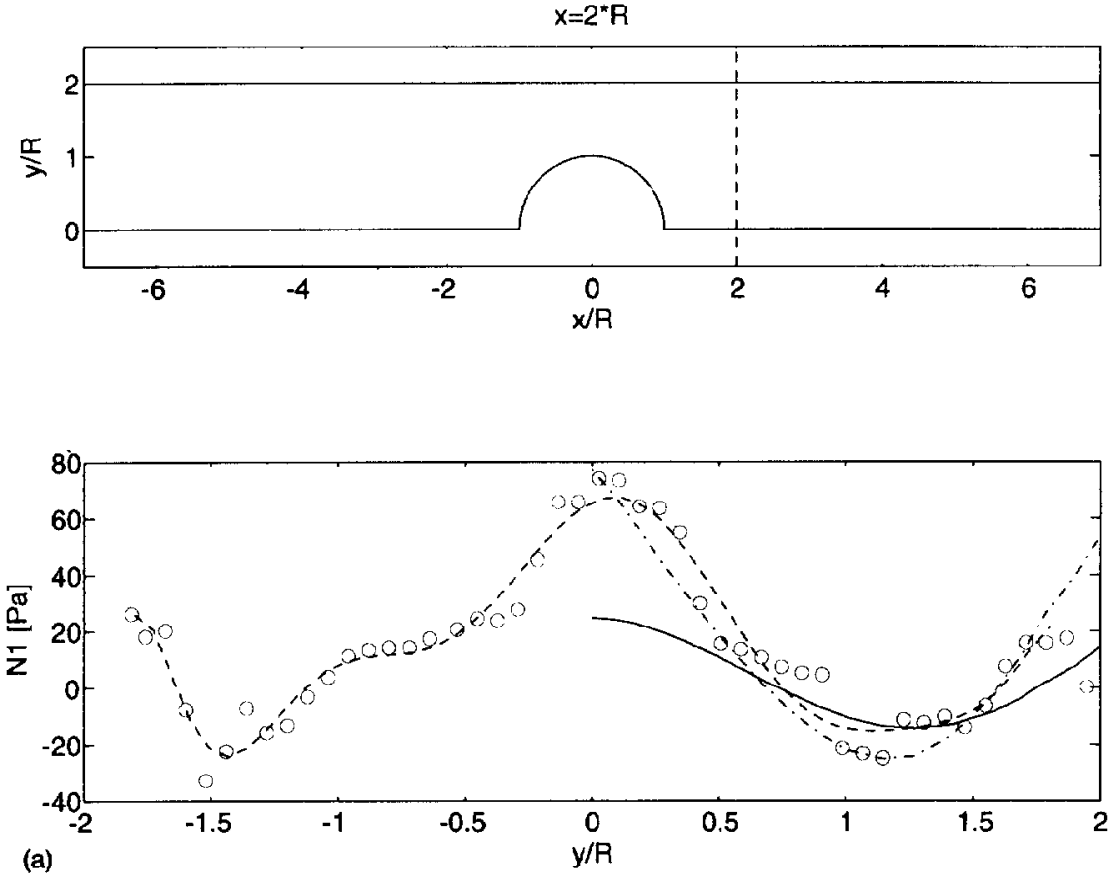

$x=3.5^{\star} R$
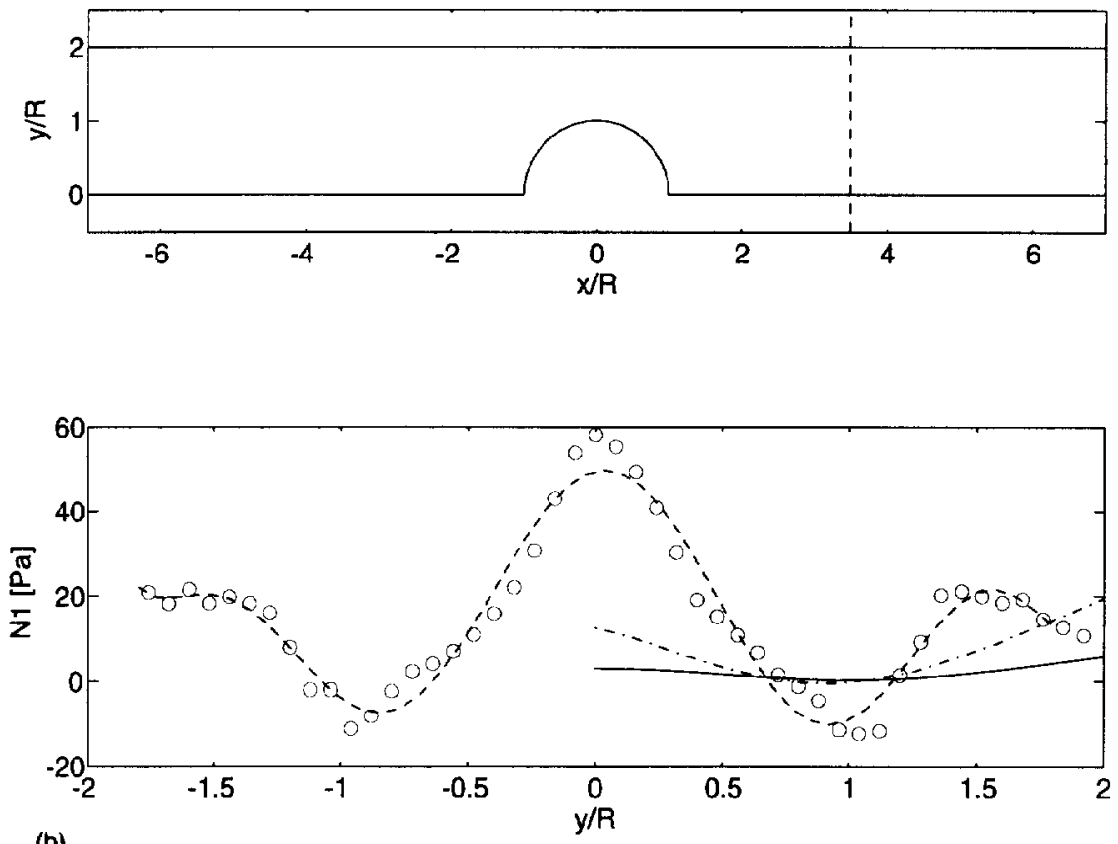

(b)

FIG. 9. Comparison of the first normal stress difference $N_{1}$ at (a) $x=2 R$, (b) $x=3.5 R$, and (c) $x=5 R$. The dashed line represents a polynomial fit through the data points indicated by the open symbols. The solid lines represent the computed results based on the EUT-fit parameters, and the dash-dotted lines are based on the MIT-fit parameters. 

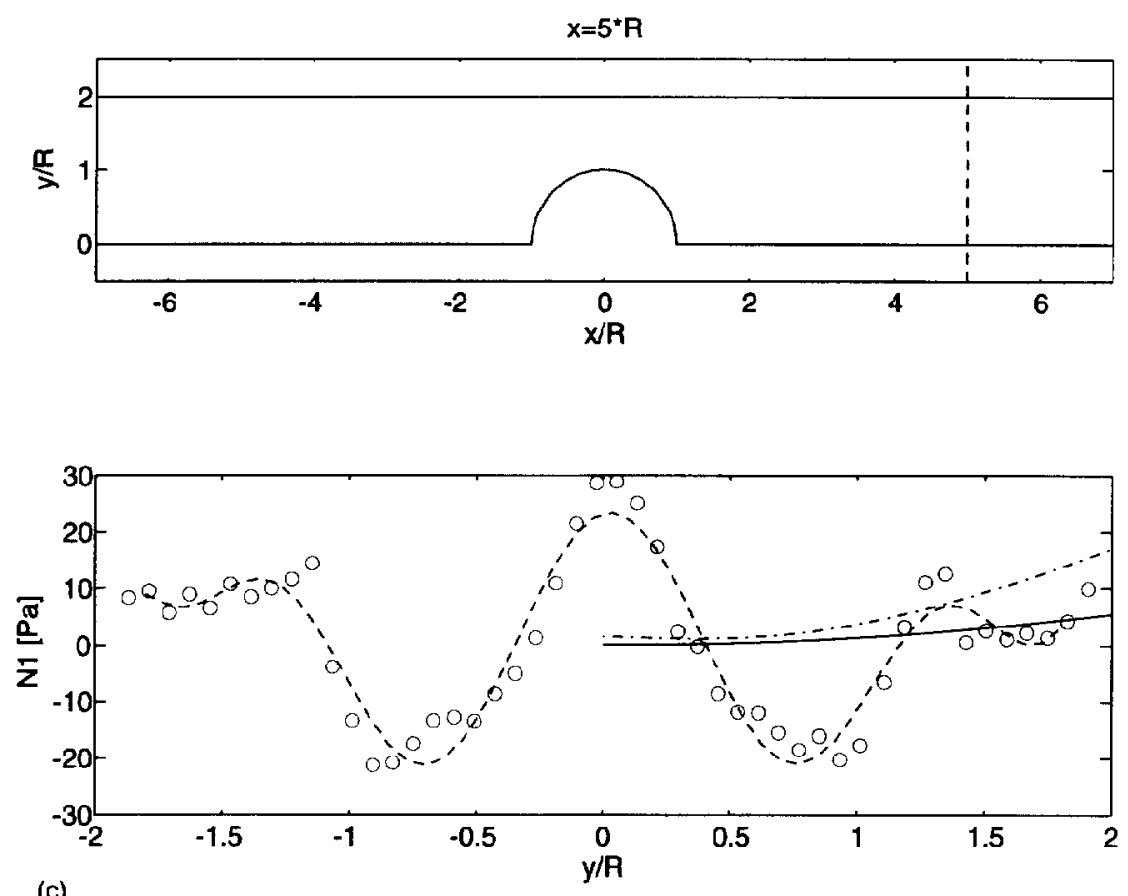

(c)

FIG. 9. (Continued.)

is found at the wake of the cylinder, while before the cylinder good agreement is found. This clearly indicates the failure of the Phan-Thien Tanner model to capture the complicated stress structure for the flow around a cylinder. Furthermore, large differences are found between the various parameter fits.

From Fig. 7, it can be seen that the shear stress is predicted quite well at $x / R=0$ by the EUT-fit parameters, but that the first normal stress difference prediction is off by at least $50 \%$. In all cases with $x / R \geqslant 2$, the first normal stress difference is predicted poorly.

The MIT-fit parameters appear to do a better job, but also fail completely at $x / R \geqslant 3$, as can be seen from Figs. 9 and 10. But these results suggest that the relaxation time should be much larger than what is expected from viscometric measurements. To illustrate this, a range of relaxation times is used in conjunction with the MIT-fit parameters for $\epsilon, \eta$, and $\eta_{0}: \lambda=0.02,0.04,0.06,0.08$, and 0.1 . The effect on $N_{1}$ is shown in Fig. 13. In particular, for $x / R \leqslant 3$ results improve with increasing relaxation time, but, still, at $x / R=5$ little change is found, which is also clearly illustrated by Fig. 13(c). In conclusion, increasing the relaxation time improves the initial magnitude of the stresses somewhat, but does not affect the stress relaxation significantly compared to the measured data.

To investigate the elongational behavior behind the cylinder in somewhat more detail, computed and measured velocities, elongational rate, and first normal stress difference are compared in Fig. 14. In the top plate, solid lines represent computed FEM values based on EUT-fit parameters. The dashed line in the velocity plot is obtained, based on a polynomial fit of the measured velocities, indicated by the open circles. 

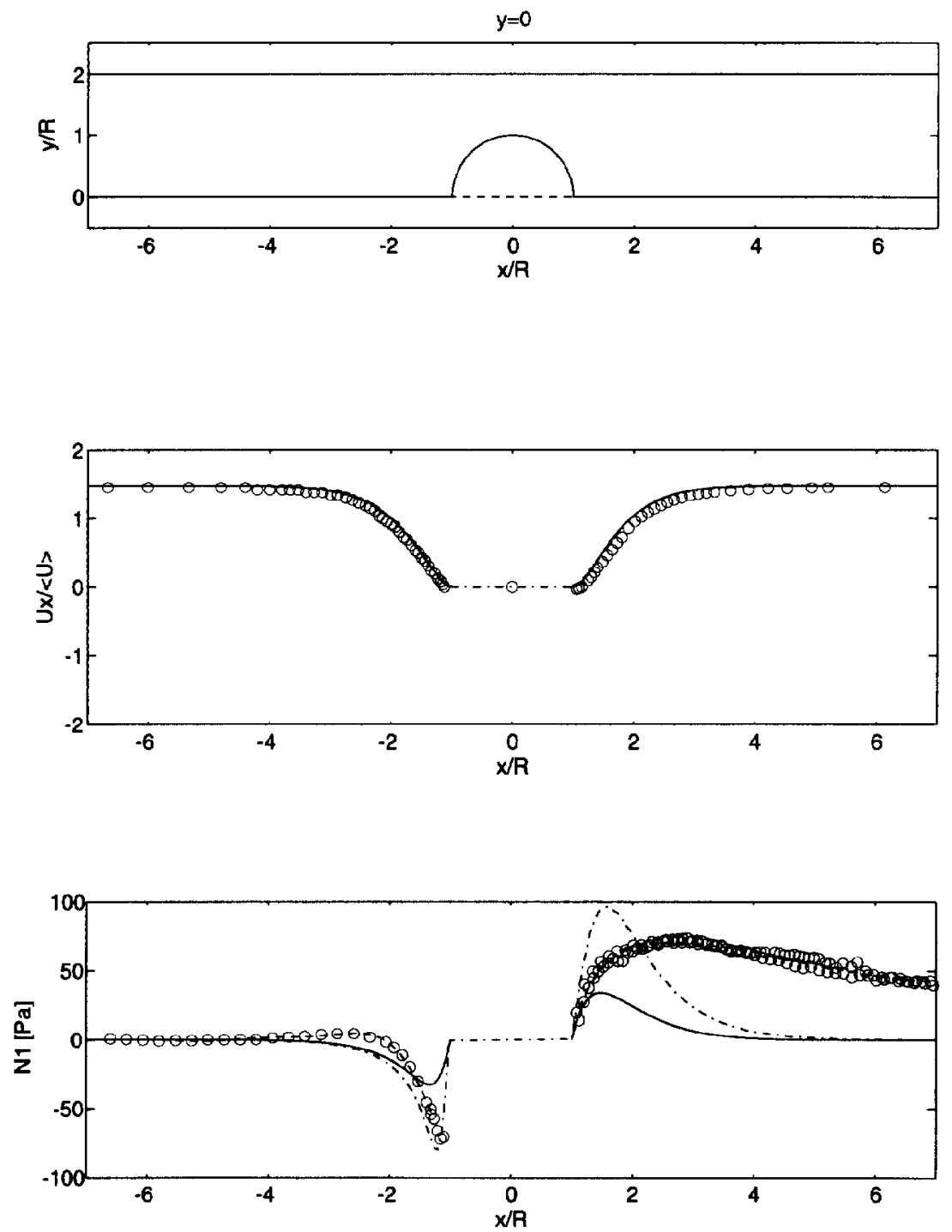

FIG. 10. Comparison of velocity $u_{x}$ and first normal stress difference $N_{1}$ along the symmetry line.

Based on this fit the elongational rate is computed and compared with the FEM results in the second plate. In the bottom plate, this "experimental" elongational rate is used to compute the first normal stress difference, both with the EUT-fit parameters (dash-dotted line) and the MIT-fit parameters (dotted line). The solid line is obtained from the FEM computed with the EUT-fit parameters. The four-mode fit of Armstrong et al. (1992) (see Table II) gives the dashed line in the bottom figure.

The open circles, again, represent the measured results. The tremendously slow relaxation of $N_{1}$ cannot be explained. There is not a significant difference between measured and computed elongational rate and, consequently, computed FEM stresses differ little from the stresses computed based on the measured elongational rate. Fur- 


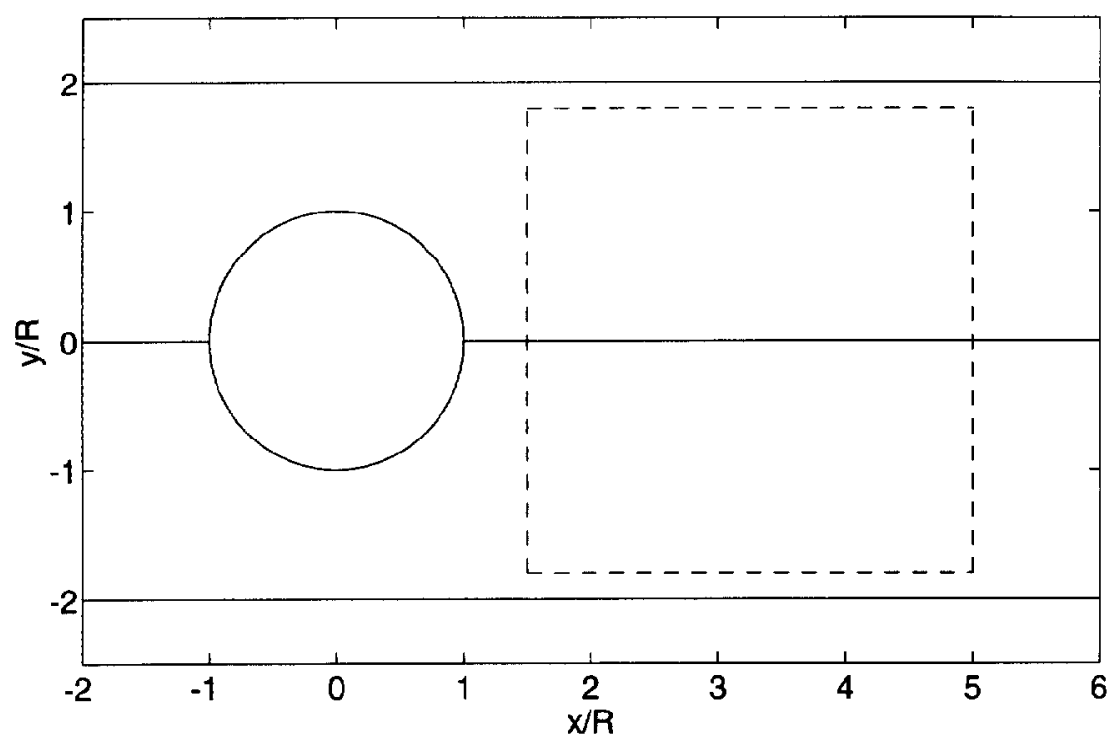

(a)

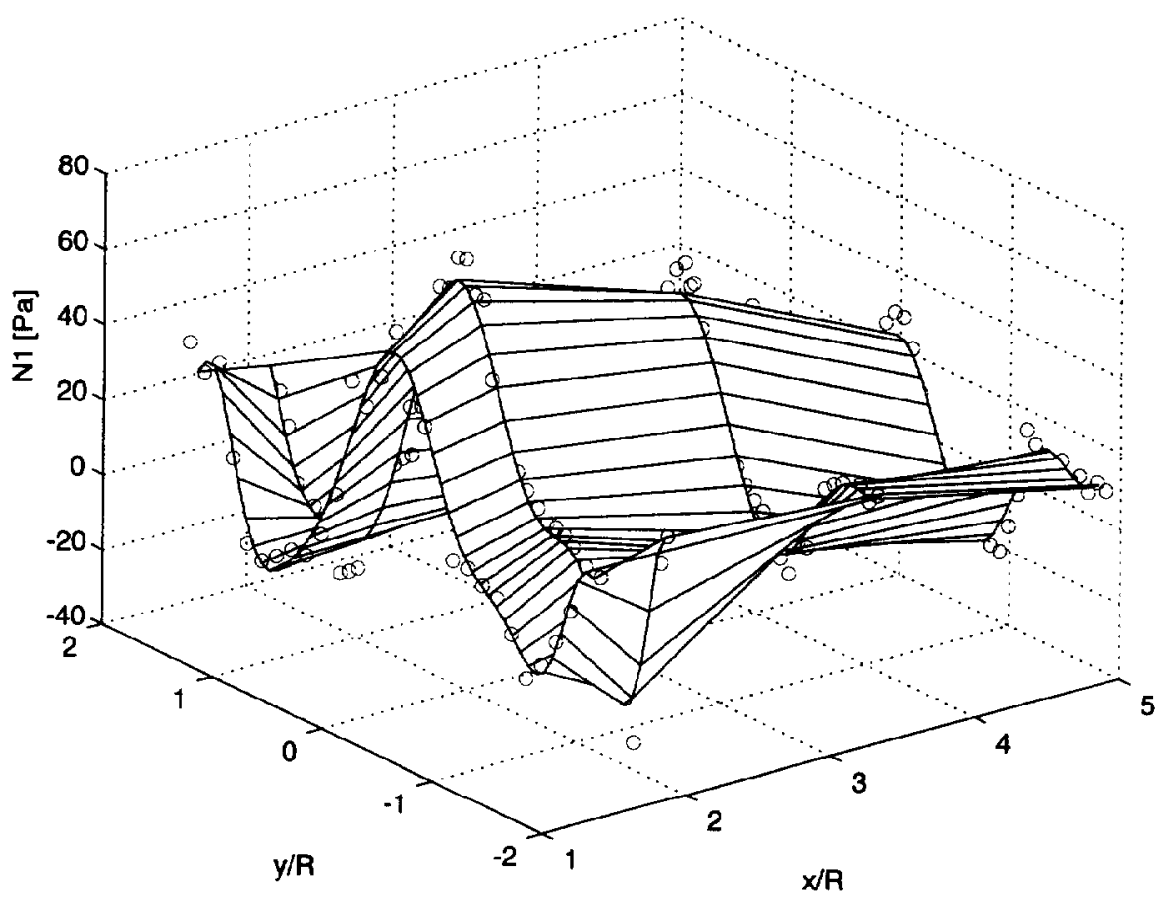

(b)

FIG. 11. (a) The flow domain considered in plate (b) and (c) $x / R \in[1.5,5], y / r=[-1.8,1.8]$; (b) measured distribution of $N_{1}$ behind the cylinder; and (c) computed distribution of $N_{1}$ behind the cylinder. The flow is in the positive $x$ direction. 


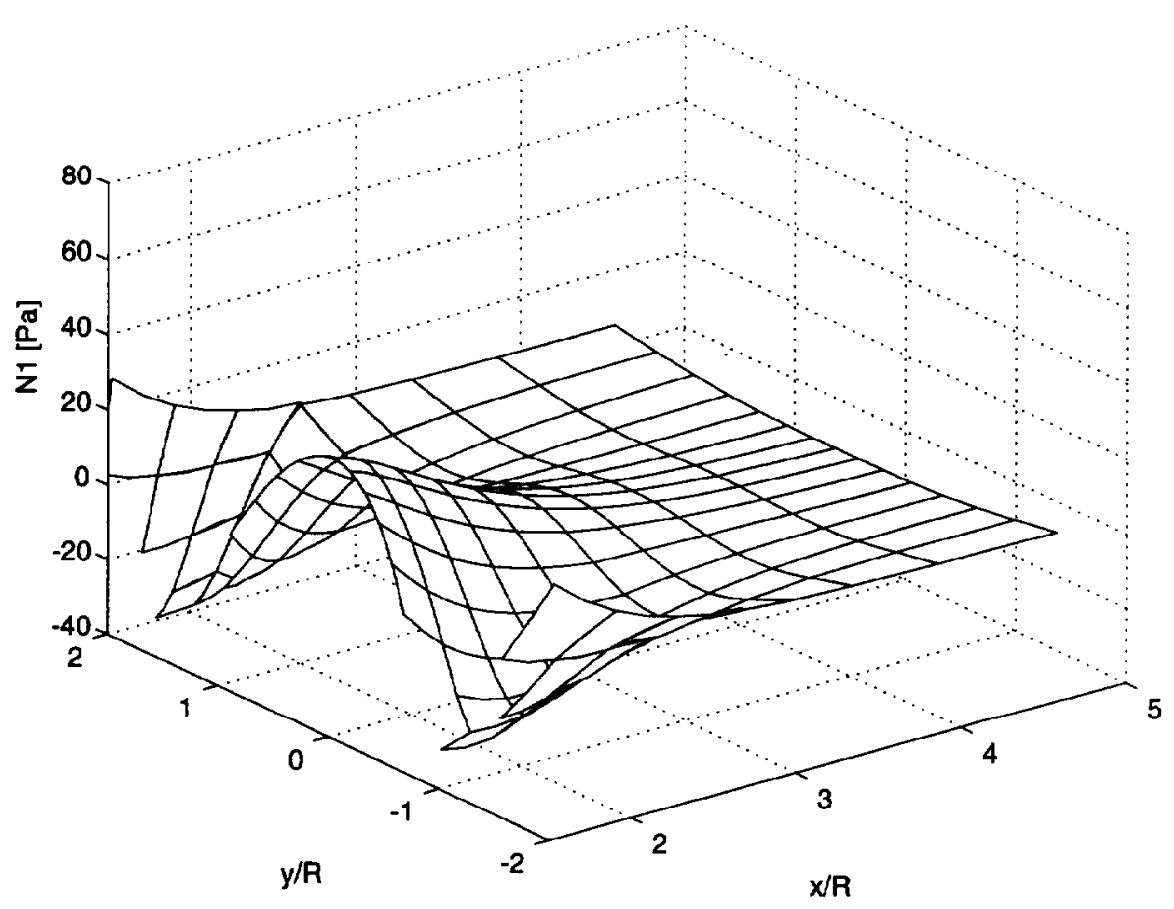

(c)

FIG. 11. (Continued.)

thermore, the use of four-mode relaxation time spectrum, giving a better quantitative fit of the experimental shear data, produces only marginally better results compared to the one-mode fit. This is in agreement with results for the four-to-one contraction, as documented in Baaijens (1993). Studying particles that enter the flow domain just above the symmetry line using particle tracking techniques, hence having a different initial condition when entering the elongational flow domain, gives the same result.

Close to the cylinder, $x / R<1.15$, the measured velocities are negative, their magnitude, however, is within the experimental error of the measuring system. Furthermore, measurements near the cylinder wall are quite difficult due to reflections of the cylinder wall. Therefore, the stress computation is started at $x / R=1.15$, assuming a zero initial condition for $N_{1}$ at this point. This explains why the dash-dotted and dotted lines in Fig. 14 take off at $x / R=1.15$ rather than at $x / R=1$, as one would expect.

Finally, a parameter sensitivity study is performed based on the "experimental" elongational rate at the wake of the cylinder. The effect of relaxation time on the stress field is demonstrated before, e.g., Fig. 13(a). Now, the nonlinearity parameter $\epsilon$ is varied and an additional parameter is introduced: $\xi \in[0,1]$, used to obtain a combination of the upper and lower convected derivative of the extra stress tensor, giving the Gordon-Schowalter derivative. Hence, the objective derivative of $\tau$ in the PTT model is assumed to be given by

$$
\boldsymbol{\nabla}=(1-\xi)\left(\frac{D \tau}{D t}-\mathbf{L} \cdot \boldsymbol{\nabla}-\tau \cdot \mathbf{L}^{c}\right)+\xi\left(\frac{D \tau}{D t}+\mathbf{L} \cdot \tau+\tau \cdot \mathbf{L}^{c}\right)
$$




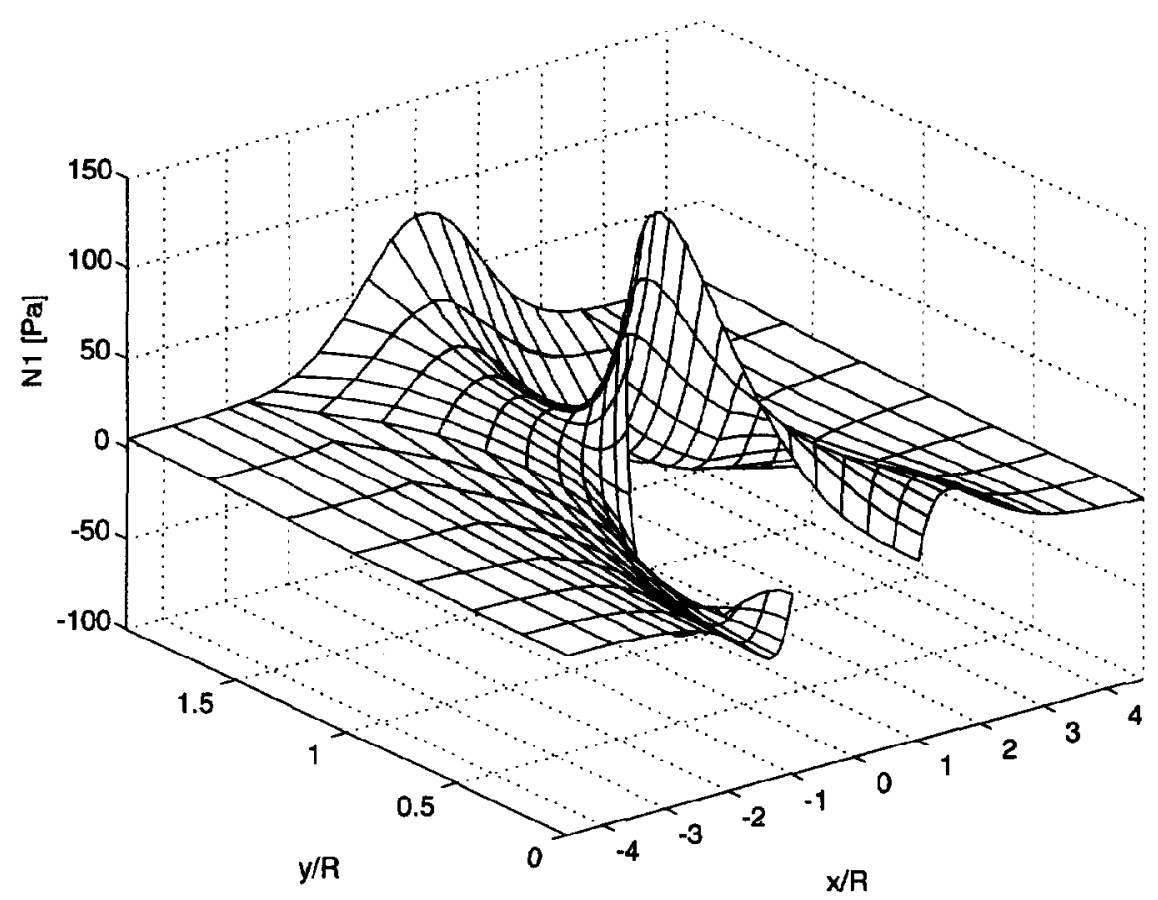

FIG. 12. Computed distribution of $N_{1}$ in the vicinity of the cylinder: $x / R \in[-4.5,4.5]$ and $y / R \in[0,2]$. Notice that, compared to the previous two figures, only the positive $y / R$ domain is shown, while a larger $x$ range is considered. The center of the cylinder is located at $(x / R, y / R)=(0,0)$. The flow is in the positive $x$ direction.

Using the MIT-fit parameters with respect to fixed variables, Fig. 15 shows the effect of an $\epsilon$ range of $0,0.1,0.2,0.3$, and 0.4 on the first normal stress difference $N_{1}$. The open symbols represent the measured data and the solid lines the computed values. With increasing $\epsilon$, the maximum stress level decreases monotonically, as expected. Essentially, only the vertical scaling is affected and no significant change in the shape of the curves is noticed. At $\epsilon=0$ the PTT model without solvent viscosity reduces the Upper Convected Maxwell model, known to be very sensitive to elongational flows, as illustrated here as well. Changing $\epsilon$ from 0.1 to 0.4 , gives a reduction in maximum stress level of almost $50 \%$. So, the combined effect of a lower relaxation time and a higher $\epsilon$ parameter of the EUT fit accounts for the large difference between the EUT-fit and MIT-fit results.

Figure 16 demonstrates the effect of mixing the upper and lower convected derivative. The $\xi$ parameter is varied in the same range as the $\epsilon$ parameter, and the effect is likewise: increasing the $\xi$ value gives a monotonically decreasing maximum stress level. Again, only the vertical scaling is affected, and little effect on the shape of the stress curves is seen.

\section{DISCUSSION}

A striking difference is found between the computed and measured stress fields behind the cylinder at a moderate value of the Deborah number, while before the cylinder good agreement is found. This result is quite surprising, as in previous work 

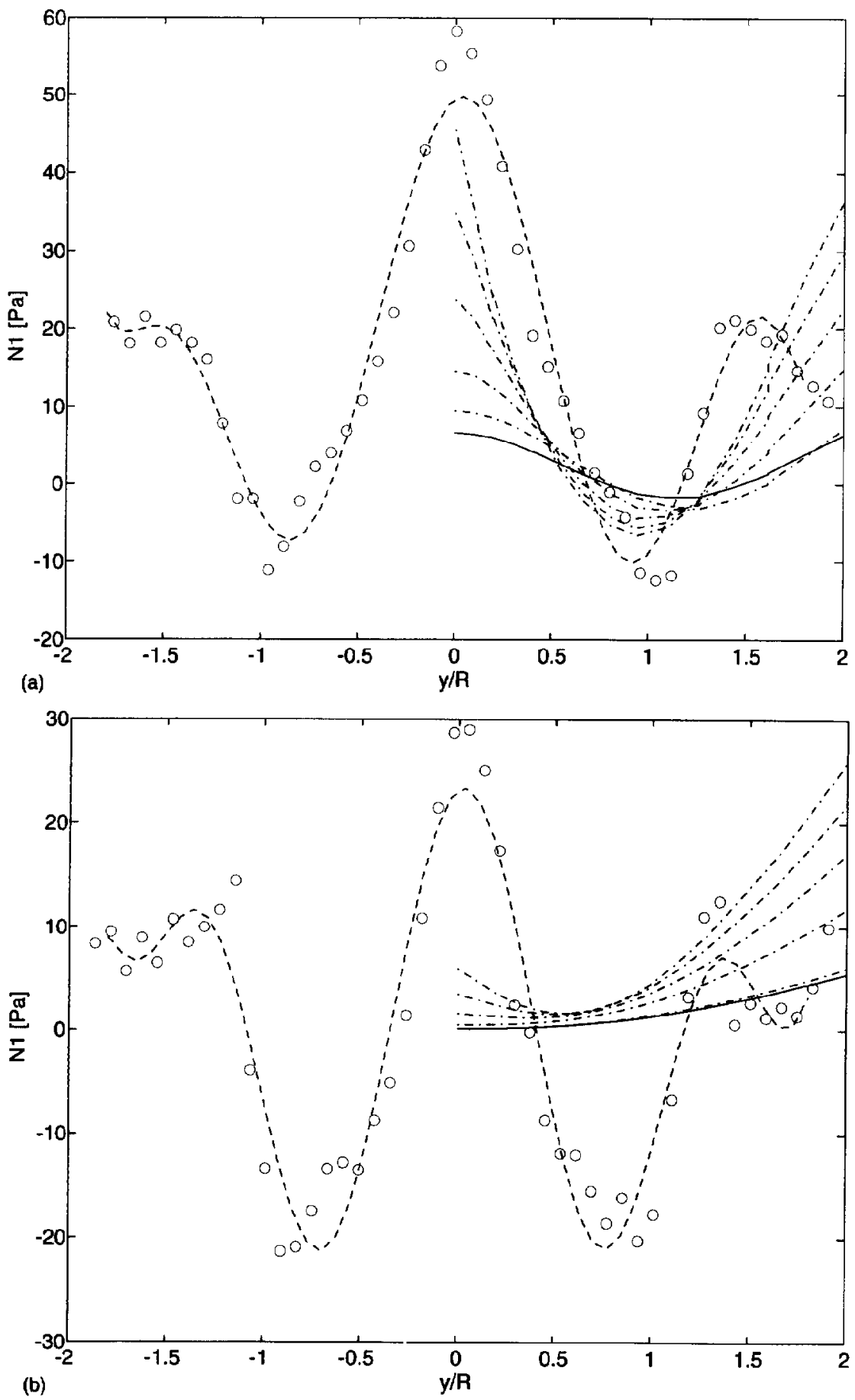

FIG. 13. Effect of relaxation time on $N_{1}$. (a) At $x / R=3$; (b) at $x / R=5$; (c) along the symmetry line. The dash-dotted lines are obtained by increasing relaxation times: $\lambda=0.02,0.04,0.06,0.08$, and 0.1 . In all cases, the first normal stress difference increases monotonically with increasing relaxation time. The open symbols denoted the measured results, the dashed line is a polynomial fit through these data points, and the solid line represents the computed profile based on the EUT-fit parameters. 


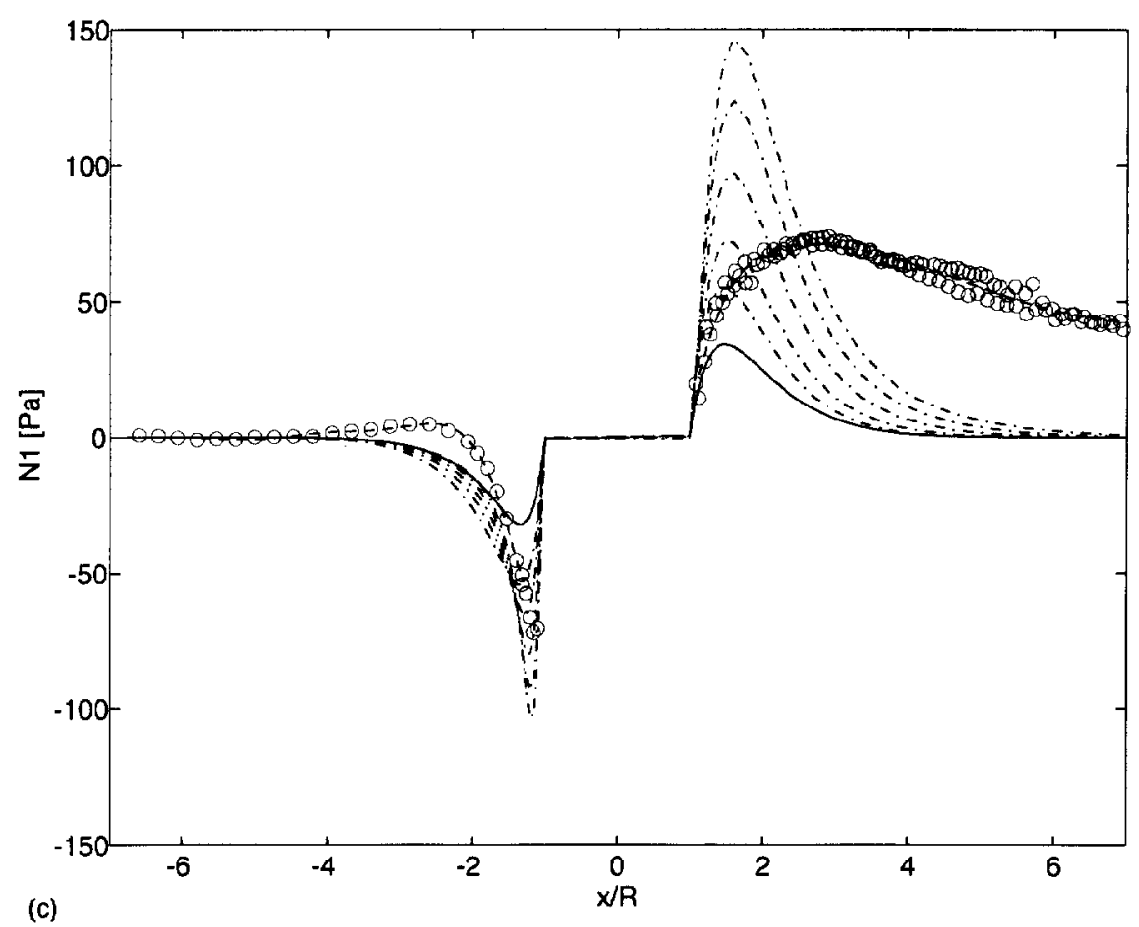

FIG. 13. (Continued.)

good qualitative and quantitative agreement was found for the flow of the PIB/C14 solution through a planar four-to-one contraction [Baaijens (1993)]. A number of possible explanations for this can be given.

In Baaijens (1993), a four-mode PTT model is used, but in that study it was also shown that the use of a one-mode PTT model did not lead to strong qualitative differences, at least away from the corner singularity. The investigations reported in Fig. 14 also demonstrate that the effect of a four-mode PTT model is marginal compared to the strong mismatch between the computed and experimental stress profile.

The stress optical coefficient was chosen according to Quinzani (1991). We could not check this value for our fluid. Yet, a different stress optical coefficient will only change the absolute value of the measured data and does not explain the qualitative differences found. Furthermore, the stress optical rule is still expected to hold, as the stress level in the wake of the cylinder is of the same order of magnitude as in the four-to-one contraction studied by Quinzani (1991).

Given the symmetry and smoothness in our measurements, the use of the somewhat too birefringent borosilicate crown glass (BK7), necessitating the two-pass measurement cycle, is not expected to be the cause of the discrepancies found. But this point certainly needs improvement.

A strong difference exists, though, from a microrheological point of view. A fluid particle entering the four-to-one contraction along or near the symmetry line, starts with a stress of about zero, and is suddenly subjected to a strong elongational flow. This also holds for fluid particles along the symmetry line in front of the cylinder. In both cases good agreement between predicted and measured stress profiles is found. A 

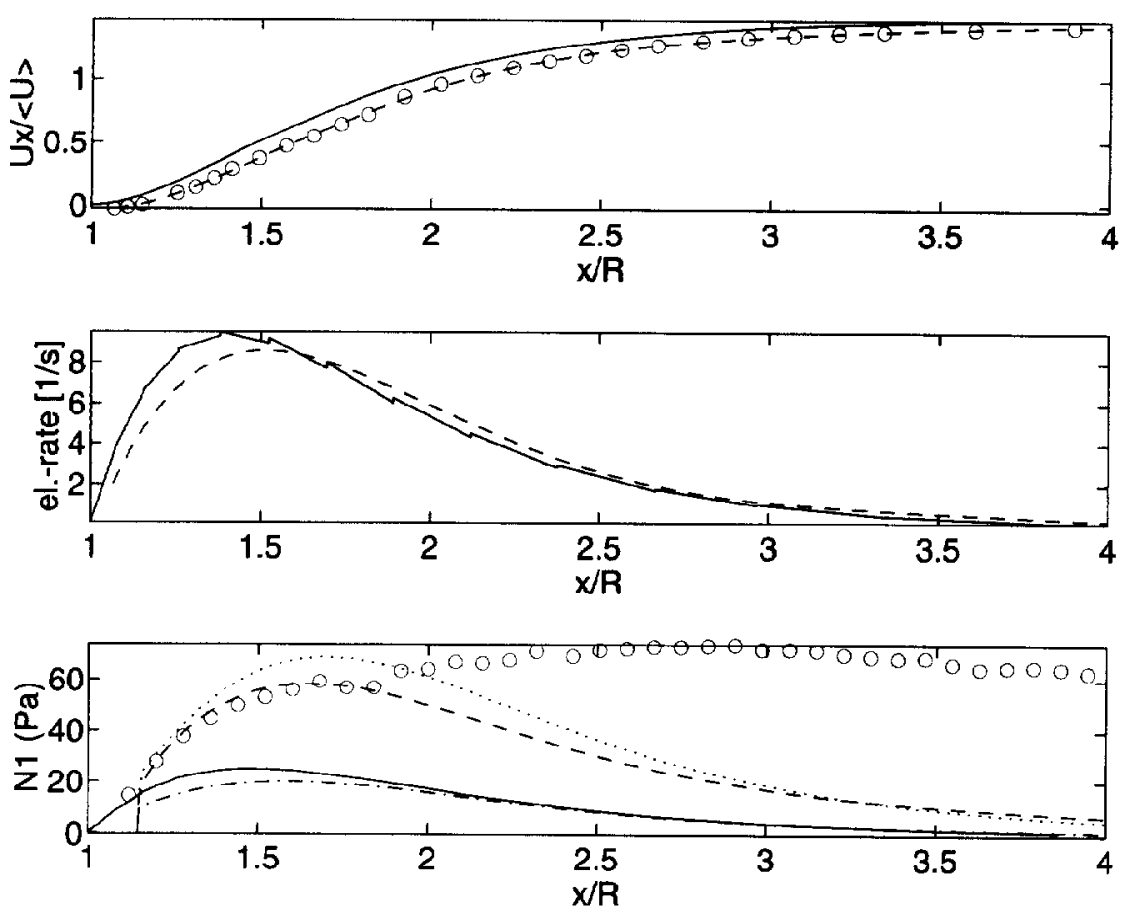

FIG. 14. Velocity, elongational rate, and first normal stress difference based on both finite element results, measured data, and polynomial fits. In the top plate, the solid line is obtained by using the EUT-fit parameters, the open symbols denote the measured data, and the dashed line is the polynomial fit of the measured data. In the middle plate, the solid line represents the computed FEM results, and the dashed line is the computed elongational rate from the polynomial fit of measured velocity data. In the bottom plate, open symbols denote the measured first normal stress difference $N_{1}$, the solid line is the computed FEM profile based on EUT-fit parameters, the dash-dotted line is the computed stress profile based on the polynomial velocity fit using the EUT-fit parameters, while the dotted line uses the MIT-fit parameters and the dashed line is based on the four-mode fit of Table II.

fluid particle that enters the elongational region behind the cylinder, though, is first subjected to a strong shear flow as it passes the cylinder. Shear rates along the cylinder wall exceed $100 \mathrm{~s}^{-1}$. Behind the cylinder, the maximum elongational rate is of order $10 \mathrm{~s}^{-1}$, which is of the same order as the elongational rate obtained in the experiments of Quinzani et al. (1991). This difference in flow history may be the cause of the discrepancies found, and appears to indicate that the PTT model does not capture such a complicated flow history accurately.

TABLE II. Four-mode linear relaxation time spectrum of Armstrong et al. (1992).

\begin{tabular}{cccc}
\hline \hline Mode & $\lambda_{i}$ & $\boldsymbol{\eta}_{i}$ & $\epsilon_{i}$ \\
\hline 1 & 0.6855 & 0.0400 & 0.1 \\
2 & 0.1396 & 0.2324 & 0.1 \\
3 & 0.0389 & 0.5664 & 0.1 \\
4 & 0.0059 & 0.5850 & 0.1 \\
(0) solvent & & 0.0020 & \\
\hline
\end{tabular}




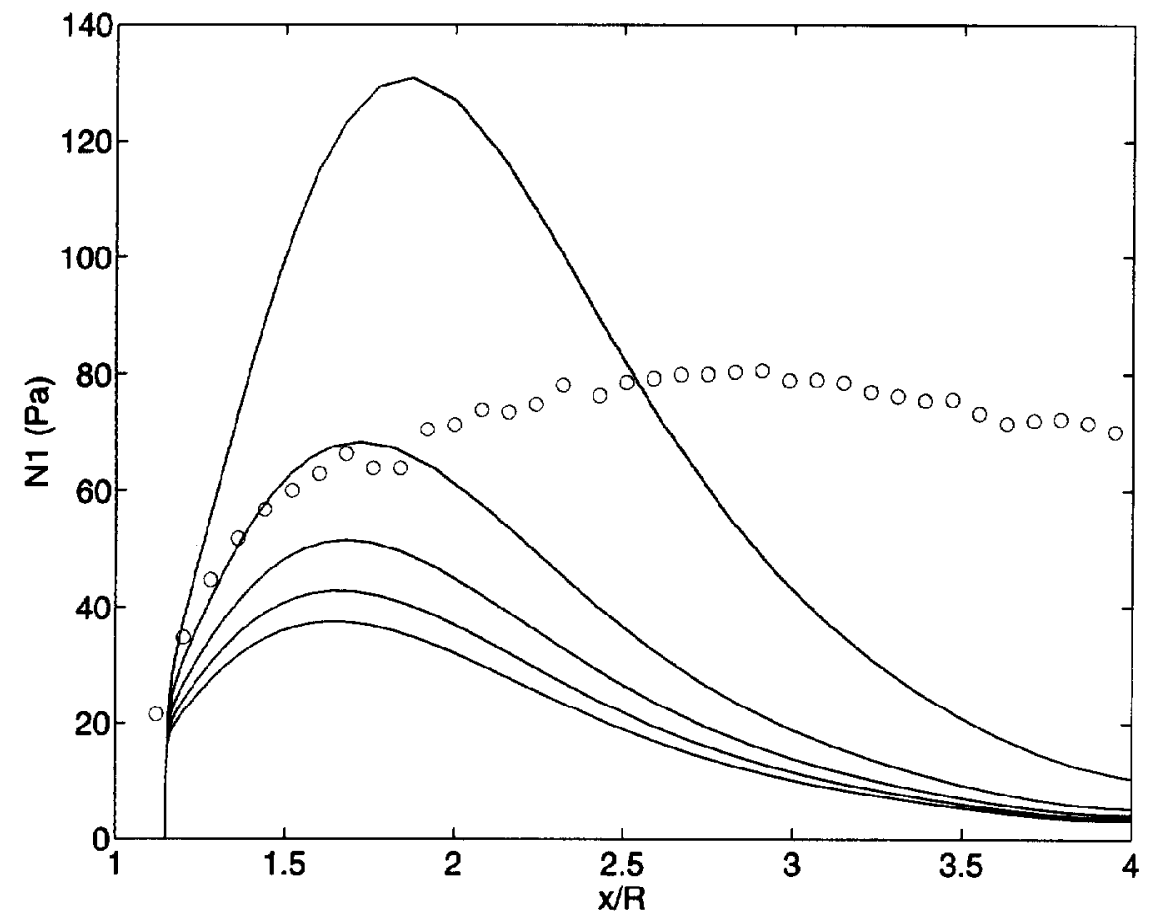

FIG. 15. Effect of changing the $\epsilon$ parameter from 0 to 0.4 in four equal steps of 0.1 on the first normal stress difference on the symmetry line at the wake of the cylinder. With increasing $\epsilon$ the maximum stress level decreases. The open symbols denote the measured first normal stress difference.

All this, despite the fact that fluid particles close to the rear stagnation points are fully relaxed, i.e., both experiments and computations indicate a vanishing stress upon approaching the rear stagnation point from the upstream direction. In the experiments, however, a laser beam of finite width ( $d=0.3 \mathrm{~mm}$, hence $d / R=0.04$ ) is used to measure birefringence. Meaning that an area-averaged value of the birefringence is obtained. So, not only particles emenating from the rear stagnation point are inspected, but also particles that have sheared along the cylinder wall. This is clearly illustrated by investigating the flow path of particles within the area of the laser beam for $x / R>3$. The dashed lines in Fig. 17 show the flow paths of two particles. At the time that they pass $x / R=10$, one is located at $y / R=0.04$; hence, at the edge of the laser beam, and one at $y / R=0.0027$. The solid line represents the cylinder wall. The dotted line represents the width of the beam when measuring along the symmetry line. From this picture it is clear that for $x / R>1.5$, virtually all particles have experienced significant preshearing when entering the elongational flow domain. Only very few particles emanating from the rear stagnation area are present.

A possible explanation for the unexpected stress distribution may be found in the theory of dumbbells with variable drag, as indicated in Sec. 8.6.2 of Larson (1988). If the coil-to-stretch transition is much faster than the stretch-to-coil transition, this might explain the very slow relaxation found in the experiments. This certainly is a topic of future investigations. 


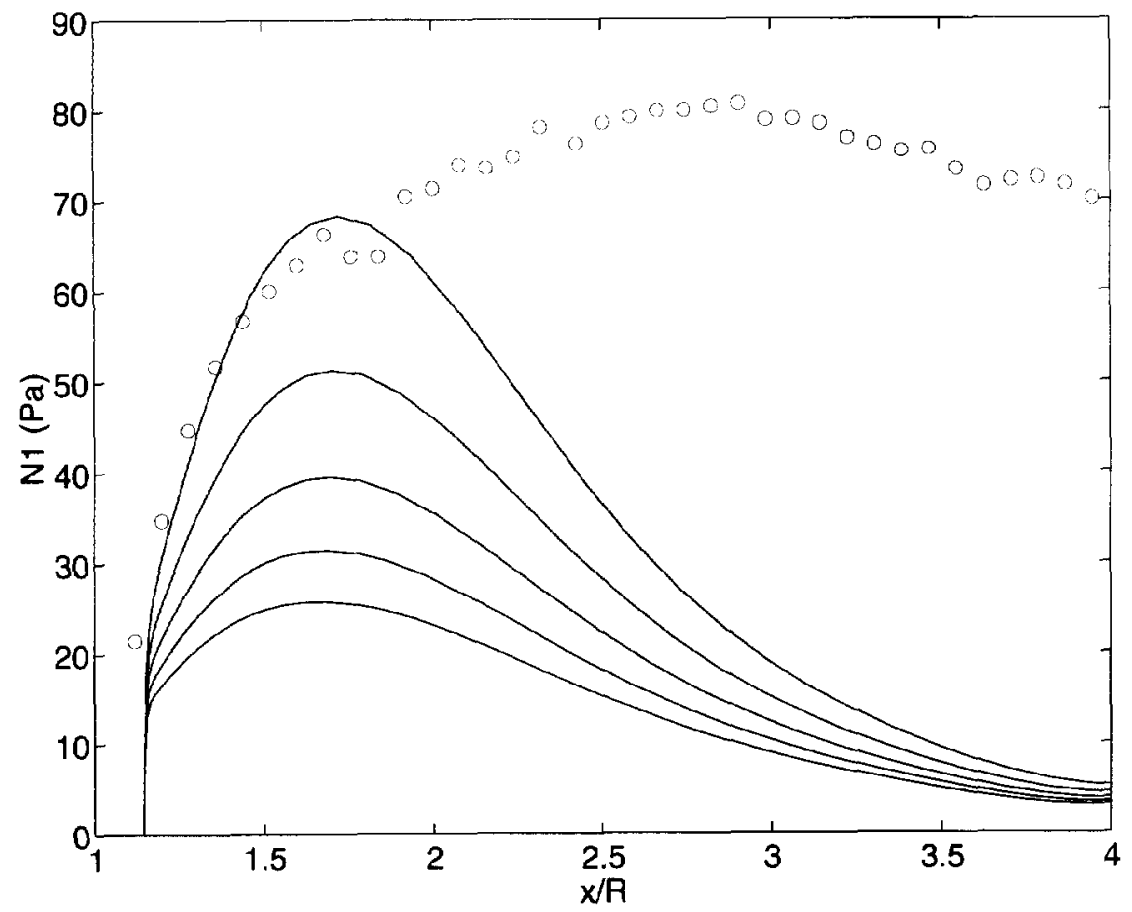

FIG. 16. The effect of changing the $\xi$ parameter, to mix the upper and lower convected derivative, from 0 to 0.4 in four equal steps of 0.1 on the first normal stress difference on the symmetry line at the wake of the cylinder. With increasing $\xi$ the maximum stress level decreases. The open symbols denote the measured first normal stress difference.

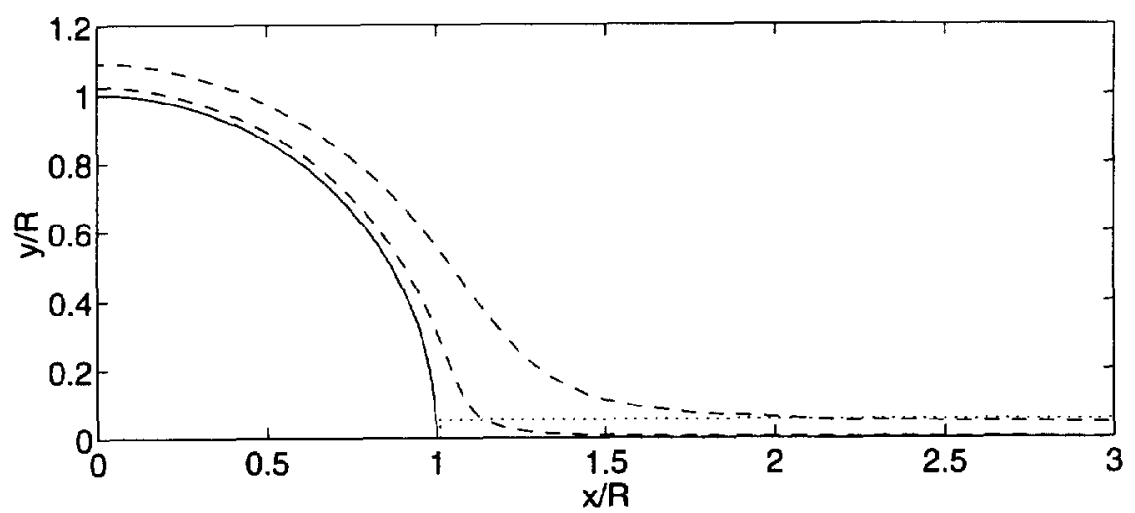

FIG. 17. Particle path of two particles. When passing $x / R=10$, one particle is at $y / R=0.04$, while the other is at $y / R=0.027$. The dashed lines represent the particle paths, the solid line the cylinder wall, and the dotted line the width of the laser beam. 
In conclusion, combined stress-optical and velocity measurements are a powerful tool to examine the performance of constitutive models in complex multidimensional flows, and may provide guidelines to design improved constitutive models. Furthermore, it is demonstrated that constitutive parameters obtained from fitting simple viscometric flow data is insufficient to predict complex rheological flows. Hence, based on current results, the conjecture made in the Introduction that parameter estimation using complex, multidimensional flows is essential to obtain good constitutive equations and parameters, appears to hold.

Future work is directed toward improving our experimental facilities to overcome the aforementioned uncertainties and on enhancing current constitutive models to allow for a more realistic modeling of multidimensional viscoelastic flows.

Note added in proof: In a new series of experiments, using a different pump, new glass windows with very low residual birefringence (SF57) from SCHOTT), a new FIB-equipment and a newly prepared fluid, operated at higher characteristic flow rates, only small differences are found between measured and predicted first normal stress difference along the centerline at the wake of the cylinder. We intend to report on these findings in the future.

\section{ACKNOWLEDGMENTS}

We gratefully acknowledge the cooperation of Dr. M. van Gurp at DSM Research (Geleen, The Netherlands), who placed his FIB measurement system to our disposal, and who was of great help during the birefingence experiments. Furthermore, we gratefully acknowledge the reviewers for their helpful comments.

\section{References}

Armstrong, R. C., R. A. Brown, L. M. Quinzani, G. H. McKinley, and J. A. Bryars, "Measurement of velocity and stress fields in complex polymer flows," in Proceedings of the XI International Congress on Rheology, edited by P. Moldenears and R. Keunings (Elsevier, Amsterdam, 1992), pp. 27-32.

Baaijens, F. P. T., "Numerical analysis of start-up planar and axisymmetric contraction flows using multimode differential constitutive models, J. Non-Newt. Fluid Mech. 48, 147-180 (1993).

Chow, A. W. and G. G. Fuller, "Response of moderately concentrated xanthan gum solutions to timedependent flows using two-color flow birefringence," J. Rheol. 28, 23-43 (1984).

Drain, L. E., The Laser Doppler Technique (Wiley, New York, 1980).

Fortin, A. and M. Fortin, "A preconditioned generalized minimal residual algorithm for the numerical solution of viscoelastic flows," J. Non-Newt. Fluid Mech. 36, 227-288 (1990).

Fortin, A., A. Zine, and J-F. Agassant, "Computing viscoelastic fluid flow problems at low cost," J. Non-Newt. Fluid Mech. 42, 1-8 (1992).

Fuller, G. G., "Optical rheometry," Annu. Rev. Fluid Mech. 22, 387-417, (1990).

Fuller, G. G. and K. J. Mikkelsen, "Note: Optical rheometry using a rotary polarization modulator," J. Rheol. 33, 761-769 (1989).

Hudson, N. E. and T. E. R. Jones, "The al project, an overview," J. Non-Newt. Fluid Mech. 46, 69-88 (1993).

Larson, R. G., Constitutive Equations for Polymer Melts and Solutions (Buterworths, Boston, 1988).

Mackay, M. E. and D. V. Boger, "Rheological measurement," in Flow Visualisation in Rheometry, edited by X. Collyer and D. W. Clegg (Elsevier Applied Science, Amsterdam, 1988).

McKinley, G. H., "Nonlinear dynamics of viscoelastic flows in complex geometries," PhD thesis, MIT, Cambridge, 1991.

McKinley, G. (private communication, 1993).

Quinzani, L. M., "Birefringence studies of entry flows of concentrated polymer solutions," PhD thesis, MIT, Cambridge, 1991.

Quinzani, L. M., G. H. McKinley, R. C. Armstrong, and R. A. Brown, Modeling the rheology of polyisobutylene solutions," J. Rheol. 34, 705-749 (1990). 
Rajagopalan, D., J. A. Byars, R. C. Armstrong, R. A. Brown, J. S. Lee, and G. G. Fuller, "Comparison of numerical simulations and birefringence measurements in viscoelastic flow between rotating cylinders," J. Rheol. 36, 1349-1375 (1992).

Wales, J. L. S., "The application of flow birefringence to rheological studies of polymer melts," PhD thesis, Delft University of Technology, Delft, The Netherlands, 1976.

Walters, K., "Recent developments in rheometry," in Proceedings of the XI International Congress on Rheology, edited by P. Moldenears and R. Keunings (Elsevier, Amsterdam, 1992), pp. 16-23. 